\title{
A willow sex chromosome reveals convergent evolution of complex palindromic repeats
}

\author{
Ran Zhou', David Macaya-Sanz', Craig H. Carlson², Jeremy Schmutz ${ }^{3,4}$, Jerry W. Jenkins ${ }^{3}$, David Kudrna ${ }^{5}$, \\ Aditi Sharma ${ }^{4}$, Laura Sandor ${ }^{4}$, Shengqiang Shu ${ }^{4}$, Kerrie Barry4, Gerald A. Tuskan ${ }^{6,7}$, Tao Ma ${ }^{8}$, Jianquan Liu ${ }^{8,9}$, \\ Matthew Olson ${ }^{10}$, Lawrence B. Smart ${ }^{2}$ and Stephen P. DiFazio ${ }^{1 *}$ (I)
}

\begin{abstract}
Background: Sex chromosomes have arisen independently in a wide variety of species, yet they share common characteristics, including the presence of suppressed recombination surrounding sex determination loci. Mammalian sex chromosomes contain multiple palindromic repeats across the non-recombining region that show sequence conservation through gene conversion and contain genes that are crucial for sexual reproduction. In plants, it is not clear if palindromic repeats play a role in maintaining sequence conservation in the absence of homologous recombination.

Results: Here we present the first evidence of large palindromic structures in a plant sex chromosome, based on a highly contiguous assembly of the W chromosome of the dioecious shrub Salix purpurea. The W chromosome has an expanded number of genes due to transpositions from autosomes. It also contains two consecutive palindromes that span a region of $200 \mathrm{~kb}$, with conspicuous $20-\mathrm{kb}$ stretches of highly conserved sequences among the four arms that show evidence of gene conversion. Four genes in the palindrome are homologous to genes in the sex determination regions of the closely related genus Populus, which is located on a different chromosome. These genes show distinct, floral-biased expression patterns compared to paralogous copies on autosomes.
\end{abstract}

Conclusion: The presence of palindromes in sex chromosomes of mammals and plants highlights the intrinsic importance of these features in adaptive evolution in the absence of recombination. Convergent evolution is driving both the independent establishment of sex chromosomes as well as their fine-scale sequence structure.

Keywords: Sex, Gene conversion, W chromosome, Palindrome, Genome, Salix

\section{Introduction}

Sex chromosomes carry genes that confer or control sex-specific traits [1]. In theory, the heterogametic (sexspecific) sex chromosome evolved from an autosome. There are two important features in sex determination regions (SDRs): suppressed recombination and the presence of sequences that only occur in one sex [1]. Furthermore, many sex chromosomes have lost most of their original genes over evolutionary time and accumulated repetitive sequences such as transposable elements

\footnotetext{
* Correspondence: spdifazio@mail.wvu.edu

${ }^{1}$ Department of Biology, West Virginia University, Morgantown, WV 26506-6057, USA

Full list of author information is available at the end of the article
}

and tandem gene duplications [2, 3]. Consequently, sex chromosomes can be difficult to sequence because they are often highly heterochromatic and have a large amount of repetitive and ampliconic DNA [1, 4].

A striking characteristic of mammalian sex chromosomes is the presence of large palindromes in ampliconic regions of the $\mathrm{X}$ and $\mathrm{Y}$ chromosomes that consist of large inverted repeats with highly identical sequences that are undergoing gene conversion $[5,6]$. Ampliconic sequences on the human $\mathrm{Y}$ chromosome were acquired through transpositions from diverse sources, and then amplified [4]. These ampliconic sequences account for about $30 \%$ of the $\mathrm{Y}$ euchromatin [4]. The human Y chromosome palindromes contain eight gene families

(c) The Author(s). 2020 Open Access This article is distributed under the terms of the Creative Commons Attribution 4.0 International License (http://creativecommons.org/licenses/by/4.0/), which permits unrestricted use, distribution, and reproduction in any medium, provided you give appropriate credit to the original author(s) and the source, provide a link to the Creative Commons license, and indicate if changes were made. The Creative Commons Public Domain Dedication waiver (http://creativecommons.org/publicdomain/zero/1.0/) applies to the data made available in this article, unless otherwise stated. 
that are expressed predominantly in the testes and which are essential for spermatogenesis [6-8]. These genes undergo extensive gene conversion and have high sequence identity among the copies [6]. Other palindromes occur in the genome, but those on the sex chromosomes are by far the largest and have the highest rates of gene conversion [6,9]. Palindromes have also been found on the W chromosomes of New World sparrows and blackbirds, suggesting that this may be a widespread feature of sex chromosomes [10]. However, such structures have not yet been described in plants.

Unlike in most animals, there is a lack of obvious sex chromosome heteromorphism in most dioecious plant species (i.e., differences are not readily discernable by cytology) $[11,12]$. Sex determination systems are quite diverse in plants, and the mechanisms of sex determination have been identified for an increasing number of species in recent years [13]. For example, Y chromosomes have been intensively studied in papaya and persimmon. Both of these contain a female suppressor on the Y chromosome [13-15]. Recently, a female suppressing gene in asparagus has been identified on the $\mathrm{Y}$ chromosome using long-read sequencing technology with optical mapping [16]. Another study on octoploid strawberry found repeated transpositions of a femalespecific gene cassette [17]. The genus Silene does have clearly heteromorphic sex chromosomes and has been a long-standing model for sex determination in XY plants. Female-suppressing and male-promoting factors were identified in Silene in the 1950s using genetic approaches [18]. More recently, it has been shown that some species of Silene have ZW sex determination systems, though it remains unclear if there are commonalities in the underlying mechanisms of sex determination in XY and ZW species [19].

Sex determination is similarly diverse within the Salicaceae family. SDRs have been consistently found on chromosome 15 with female heterogamety in multiple Salix species [20-22]. This is quite different from the closely related genus Populus where sex-determining regions consistently occur on chromosome 19 , with most species showing male heterogamety [23, 24]. Previously, we reported that the SDR occupies a large portion of the W chromosome in S. purpurea with suppressed recombination extending over $\sim 5 \mathrm{Mb}[20,25]$. This is substantially larger than the SDR in $P$. trichocarpa and $P$. balsamifera, which appears to be approximately $100 \mathrm{~kb}$ in size $[24,26]$. However, due to the structural complexity of the SDRs, none of these studies have thus far included an in-depth analysis of the sequence composition and structure of the SDRs, and it is unclear whether there is a common underlying mechanism of sex determination. Here we present a much more complete assembly of the S. purpurea W chromosome and report for the first time in plants a palindromic repeat structure that is similar to the one found on mammalian Y chromosomes. We also demonstrate that gene content is expanded on the W chromosome, and homologous genes occur in the Salix and Populus SDRs, suggesting that there may be some overlap in the underlying mechanisms of sex determination in this family.

\section{Results}

\section{Genome assembly}

We present here highly contiguous genome assemblies of a female and a male $S$. purpurea. The female assembly (94006 v4) consists of 452 contigs with an N50 of 5.1 $\mathrm{Mb}$, covering a cumulative total of $317.1 \mathrm{Mb}$. Similarly, the male assembly (Fish Creek v3) has 351 contigs and an $\mathrm{N} 50$ of $5.6 \mathrm{Mb}$, covering $312.9 \mathrm{Mb}$ (Additional file 1: Table S1). Both assemblies are partially phased in genomic regions where the two haplotypes are divergent. Alternative haplotypes are represented by 421 contigs totaling $72.4 \mathrm{Mb}$ in the female assembly, and 497 contigs totaling $149 \mathrm{Mb}$ for the male. Using a genetic map from a large intercross family derived from progeny of the sequenced male genotype, we created assemblies representing the 19 chromosomes, containing 108 contigs totaling $288.3 \mathrm{Mb}$ for the female and 96 contigs totaling $288.5 \mathrm{Mb}$ for the male. These represent over $90 \%$ of the assembled sequence in both cases, though 344 and 255 contigs remained unplaced by the genetic map for the female and male, respectively (Additional file 1:Table S2). The mapped and unplaced contigs are hereafter collectively referred to as the main genome, which excludes the alternative haplotypes.

Because we expected the W haplotype to be differentiated from the $\mathrm{Z}$ haplotype in the SDR, we anticipated that much of this region would be assembled as separate contigs. These can be readily differentiated by examining the relative depth of coverage when aligning male versus female short-read sequences against these references. After identifying the location of the SDR based on the presence of sex-linked markers [20], the initial Chromosome 15 assembly appeared to consist of a mix of $\mathrm{Z}$ and W scaffolds in a region we infer to be within the SDR (Additional file 2: Figure S1a). We therefore sought to create a new assembly with $\mathrm{Z}$ and $\mathrm{W}$ haplotypes assembled to separate chromosomes. To do this, we first identified the putative $\mathrm{W}$ contigs using sex association in a population of 60 unrelated individuals and differential depth of coverage in males and females from an $\mathrm{F}_{2}$ pedigree as criteria [20]. This resulted in identifying 23 contigs that were putatively comprised primarily of sequence derived from the $\mathrm{W}$ haplotype (Additional file 1: Table S3). One scaffold was excluded because it mostly consisted of an alternative haplotype of a longer contig of Chr15W. 
Many of these contigs lacked markers from the intercross map that was used in the original genome assembly [20], particularly for those that came from portions of the $\mathrm{W}$ haplotype that were absent from the $\mathrm{Z}$ chromosome. We therefore created new genetic maps that had a mix of SNP and indel markers that would be more suited to capturing these hemizygous portions of the genome. The new genetic maps converged to 19 major linkage groups representing the 19 chromosomes. The male backcross map contained 8715 markers, while the female backcross map contained 8560 markers (Additional file 1: Table S4). We used these to assemble a $\mathrm{Z}$ and a W version of Chr15 (Additional file 1: Table S5). Thus, the current assembly (release ver5) contains 20 chromosomes, including Chr15Z and Chr15W. A total of $6.56 \mathrm{Mb}(95.7 \%)$ of the W-specific contig sequence, contained in 17 contigs, was assembled to Chr15W using these maps. Four putative W scaffolds totaling $297 \mathrm{~kb}$ in length lacked mapped markers and could not be placed unambiguously.

\section{Location of the SDR}

We repeated sex association analysis for the 60 unrelated individuals using our new assembly with Chr15Z removed. Among 54,959 tested Genotyping by Sequencing
(GBS) SNPs, all 105 significantly sex-linked SNPs were present only on Chr15W (Fig. 1a; Additional file 2: Figure S2a-c), and markers from PARs and other scaffolds in the main genome did not show any sex association (Additional file 2: Figure S2a). The eight topranking sex-associated markers were distributed from 7.66 to $8.66 \mathrm{Mb}$. Sex-associated markers were primarily heterozygous in females and homozygous in males, confirming our previously reported observation of ZW sex determination in S. purpurea [20].

\section{Composition of chromosomes $15 \mathrm{~W}$ and $15 \mathrm{Z}$}

Chr15W is $15.7 \mathrm{Mb}$ in length, composed of 22 contigs placed with the new genetic map. For comparison, Chr15Z is only $13.3 \mathrm{Mb}$ and is comprised of 16 contigs (Additional file 1: Table S5; Fig. 1). There are two pseudoautosomal regions (PARs), one at each end of Chr15W, that are indistinguishable from the corresponding regions on Chr15Z. PAR1 is $2.3 \mathrm{Mb}$ long and is composed of one contig, and PAR2 is $6.5 \mathrm{Mb}$ and is comprised of three contigs (Fig. 1). These regions are unphased and are therefore identical in the two assemblies.

The W-linked sex-determining region (SDR) is $6.8 \mathrm{Mb}$ in length and occupies nearly $40 \%$ of the chromosome (hereafter referred to as the W-SDR). This region

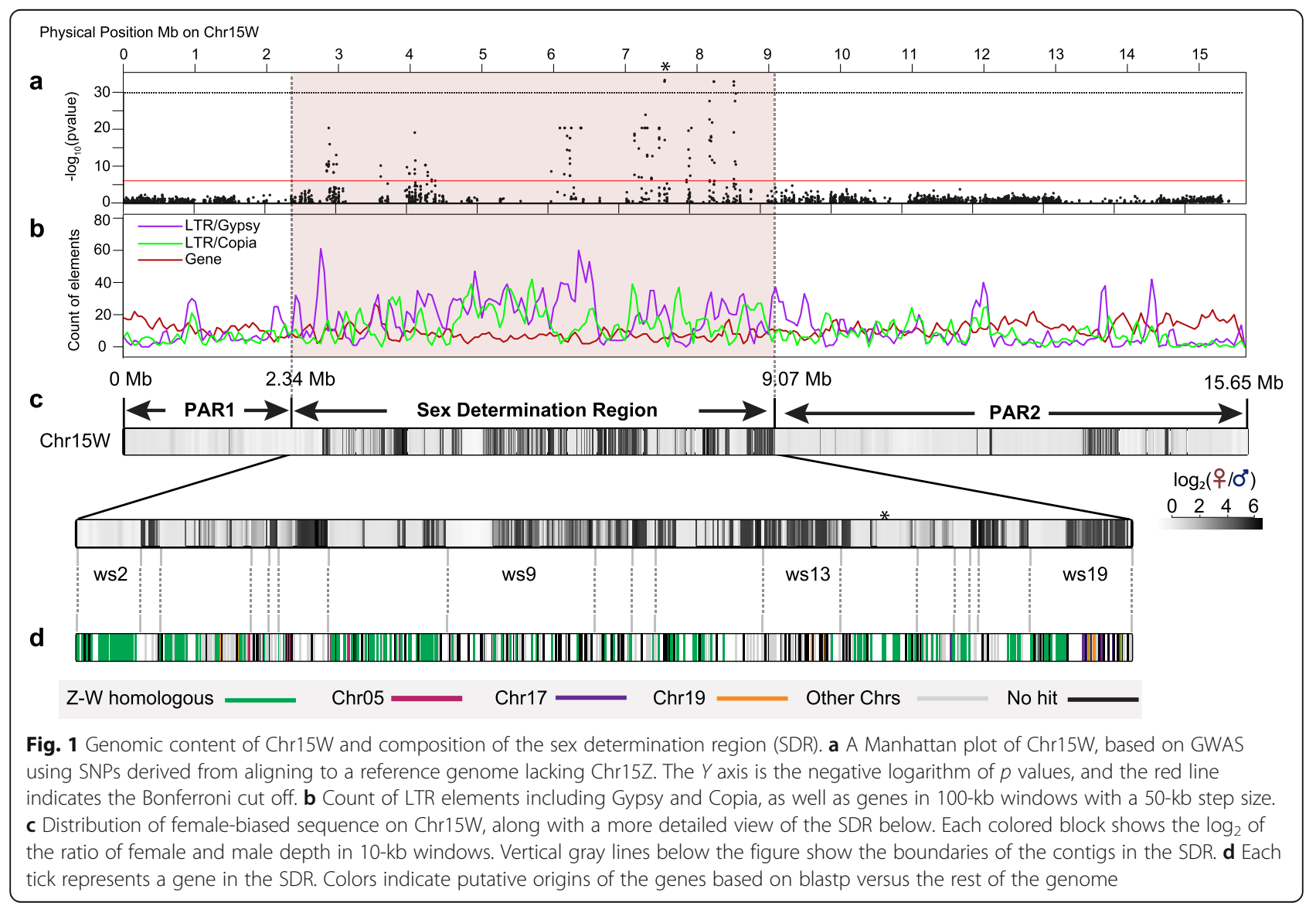


undergoes minimal recombination in the mapping population (Additional file 2: Figure S4). Reexamining male and female depth of coverage of the W-SDR, it is clear that this region of the genome is mostly phased to separate the male and female haplotypes (Additional file 2: Figure S1b). The region corresponding to the W-SDR on Chr15Z is only about $4 \mathrm{Mb}$ in length, and only occupies $28.2 \%$ of the chromosome (hereafter referred to as the Z-SDR) (Additional file 2: Figure S3). Based on the ratio of male and female depth of coverage, the $\mathrm{Z}-\mathrm{W}$ homologous regions that are present on both the $\mathrm{Z}$ and $\mathrm{W}$ chromosome are about $3.5 \mathrm{Mb}$ and insertions that are unique to the $\mathrm{W}$ are about $3.1 \mathrm{Mb}$ in the W-SDR (Fig. 1c).

The W-SDR has lower gene density and higher repeat density than other portions of the genome, suggesting that repetitive elements have accumulated in this region (Table 1). More specifically, both the W-SDR and the ZSDR show lower gene density on average than the PARs or other autosomes. Similarly, both the W-SDR and ZSDR show higher accumulation of Gypsy retrotransposons. Interestingly, Copia-LTRs occur at higher density in the W-SDR region compared to the Z-SDR $(10.9 \%$ of WSDR vs $5.9 \%$ of Z-SDR), (Kruskall-Wallis test, $P<2.2 \mathrm{e}-16$ ) (Table 1), suggesting that these inserted following cessation of recombination between these haplotypes.

\section{Gene content of the W chromosome}

There are 269 genes in PAR1, 778 genes in PAR2, and 488 genes in the W-SDR. In contrast, the Z-SDR only contains 317 genes (Fig. 2; Additional file 1: Table S6S7). An additional 29 genes are present on scaffold_844, which is likely derived from the $\mathrm{Z}$ haplotype, but which lacked genetic markers to properly place it. To evaluate the completeness of the $\mathrm{Z}$ chromosome, we compared the gene content of this region to that from the Fish Creek male reference genome. The Z-SDR region was comprised of four contigs spanning from 2.86 to 7.10 $\mathrm{Mb}$ in Fish Creek, containing a total of 333 genes. Since the size and gene content were very similar between the $\mathrm{Z}$ chromosomes of the male and female references, we

Table 1 Cumulative size in megabase of genes and LTR retrotransposons in different areas of the genome. Numbers in parentheses are percentages of the proportion of the specific type of regions

\begin{tabular}{lllll}
\hline Category & W-SDR & Z-SDR & PAR & Autosomes* $^{*}$ \\
\hline Genes & $1.56(23.8)$ & $1.14(26.8)$ & $3.72(41.9)$ & $104.31(38.1)$ \\
Total repeats & $3.16(48.1)$ & $1.81(42.4)$ & $2.58(29.0)$ & $89.17(32.6)$ \\
Gypsy-LTR & $0.86(13.2)$ & $0.55(12.8)$ & $0.38(4.3)$ & $15.45(5.6)$ \\
Copia-LTR & $0.72(10.9)$ & $0.25(5.9)$ & $0.37(4.1)$ & $13.87(5.1)$ \\
\hline
\end{tabular}

*All 18 chromosomes are included are restricting our analysis to the female to simplify the comparison.

There were 156 single-copy mutual best hits between the W-SDR and Z-SDR, referred to hereafter as Z-W homologs (analogous to $\mathrm{X}$-degenerate genes on mammalian sex chromosomes) (Fig. 2). The W-SDR also contains 32 genes in tandem duplications, while the corresponding tandem repeats in the Z-SDR contain 56 genes. Additionally, the W-SDR contains 40 genes that have mutual best hits on other autosomes, and 33 of these are tandemly duplicated in the SDR. In contrast, the Z-SDR region contains only 11 such genes, only six of which are tandemly duplicated. These putatively transposed genes comprise $8 \%$ of the W-SDR and only $3 \%$ of the Z-SDR. Another 54 genes in the W-SDR resulted from intrachromosomal transpositions and subsequent tandem duplication, while only 7 genes in this category are found on the Z-SDR. In total, these transposed and ampliconic genes account for more than half of the discrepancy in gene content between the haplotypes. An additional 103 genes in the W-SDR had a top hit to other genes in the genome, but the best hit was not mutual, so these are lower confidence candidates for transpositions or Z-W homologs. The Z-SDR contained 54 such genes. The remaining genes had no significant hits to other genes in the genome, presumably due to loss by deletion, or gaps in the sequence or annotation (85 in the W-SDR and 42 in the Z-SDR).

\section{Z-W homologs and strata}

We used syntenic gene pairs identified through MCScanX between the W-SDR and Z-SDR to test if there are strata with different degrees of divergence based on synonymous substitutions $\left(d_{S}\right)$, which would indicate different phases of cessation of recombination [27]. There was little evidence to support the presence of strata based on 156 pairs of Z-W homologs (Fig. 3 and Additional file 1: Table S8). The average $d_{S}$ was $0.027 \pm 0.020 \mathrm{SE}$. For comparison, the $d_{\mathrm{S}}$ between syntenic genes on Chr01 for S. purpurea and S. suchowensis was $0.045 \pm 0.0022 \mathrm{SE}$, and the $d_{\mathrm{S}}$ between $S$. purpurea and $P$. trichocarpa was $0.146 \pm 0.0022$ SE for syntenic genes on Chr01 (Fig. 3).

\section{Transpositions to the W-SDR and palindromic repeats}

The recently transposed genes are of particular interest because they could provide a potential mechanism for establishment of the SDR and could highlight genes that are potential candidates for sex determination and/or sex antagonism [28]. Among 40 genes putatively transposed from autosomes to the W-SDR, 7 have best hits on Chr19 (manually annotated genes excluded) (Additional file 1: Table S9). Contig ws19 is particularly enriched for transposed genes and merits a closer 


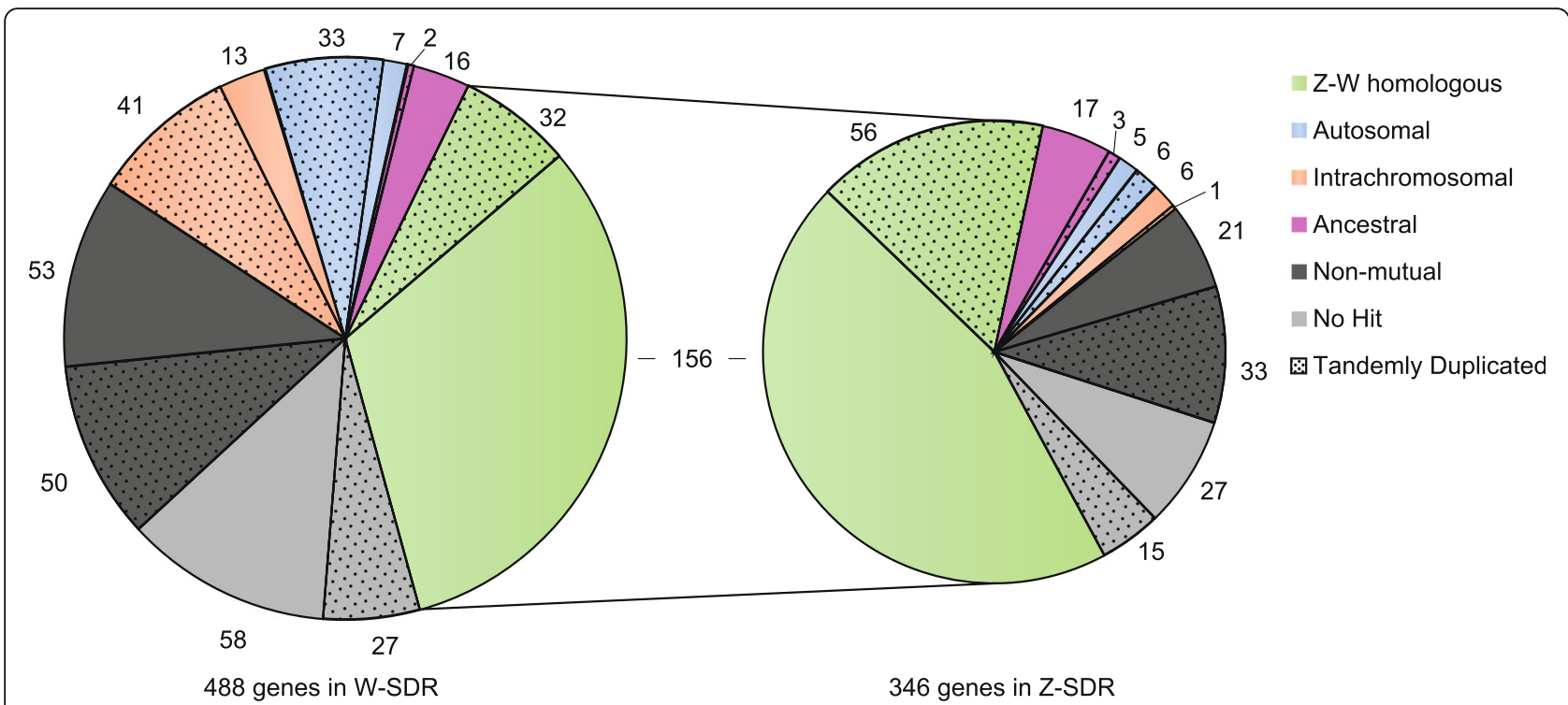

Fig. 2 Annotated genes in Chr15W and Chr15Z. Genes are grouped according to the best non-self-hit in the annotated genome. Twenty-nine genes from an unmapped Z, scaffold_844 are also included. Stippled areas indicate genes of groups identified as tandem duplicates

examination (Fig. 1). Contig ws19 contains 11 transposed genes, including four genes from Chr19 and four genes from Chr17 (Fig. 1). Many of these transposed genes occur in two to four copies on ws19 in striking inverted repeat configurations that are similar to the palindromic repeats that occur on mammalian $\mathrm{Y}$ chromosomes (Fig. 4).

In S. purpurea, this region is female-specific (i.e., it occurs in all females but in no males) and is composed of two palindromes. Palindrome W.P1 spans about $42.7 \mathrm{~kb}$

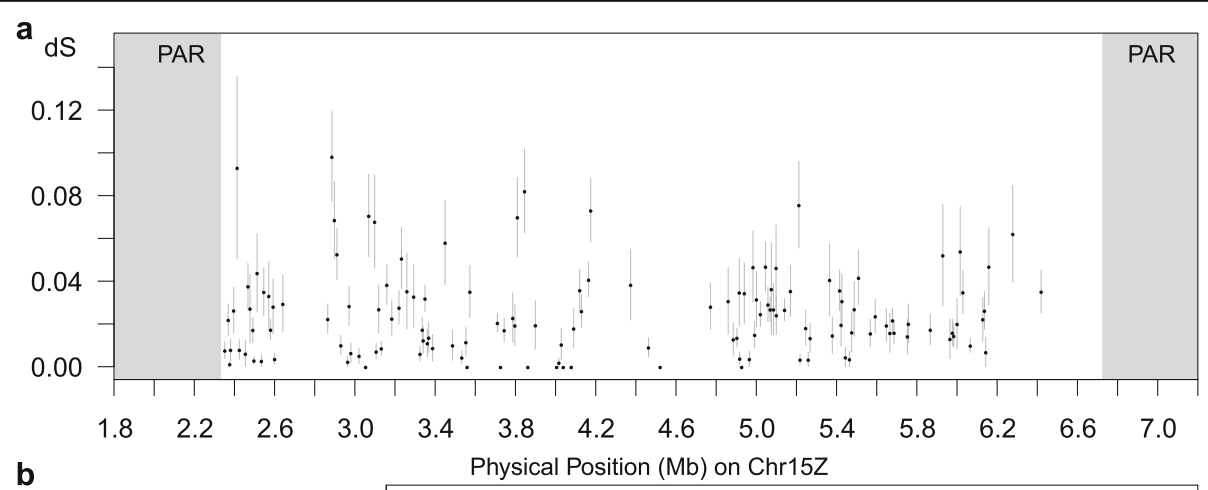

S.purpurea vs S. suchowensis

S. purpurea W vs Z

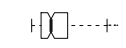

0.0

0.2

0.4
dS

0.6

0.8

Fig. 3 Synonymous substitution rates $\left(d_{S}\right)$ for genes in the SDR. a Comparison of syntenic genes in the W-SDR and Z-SDR. Bars represent standard errors. b Boxplot showing distributions of interspecific synonymous substitutions for 1365 syntenic genes on Chr01 for the closely related species S. purpurea and S. suchowensis and for 1363 genes on Chr01 in S. purpurea and Populus trichocarpa, compared to the distribution of substitutions between syntenic genes in the S. purpurea SDR 

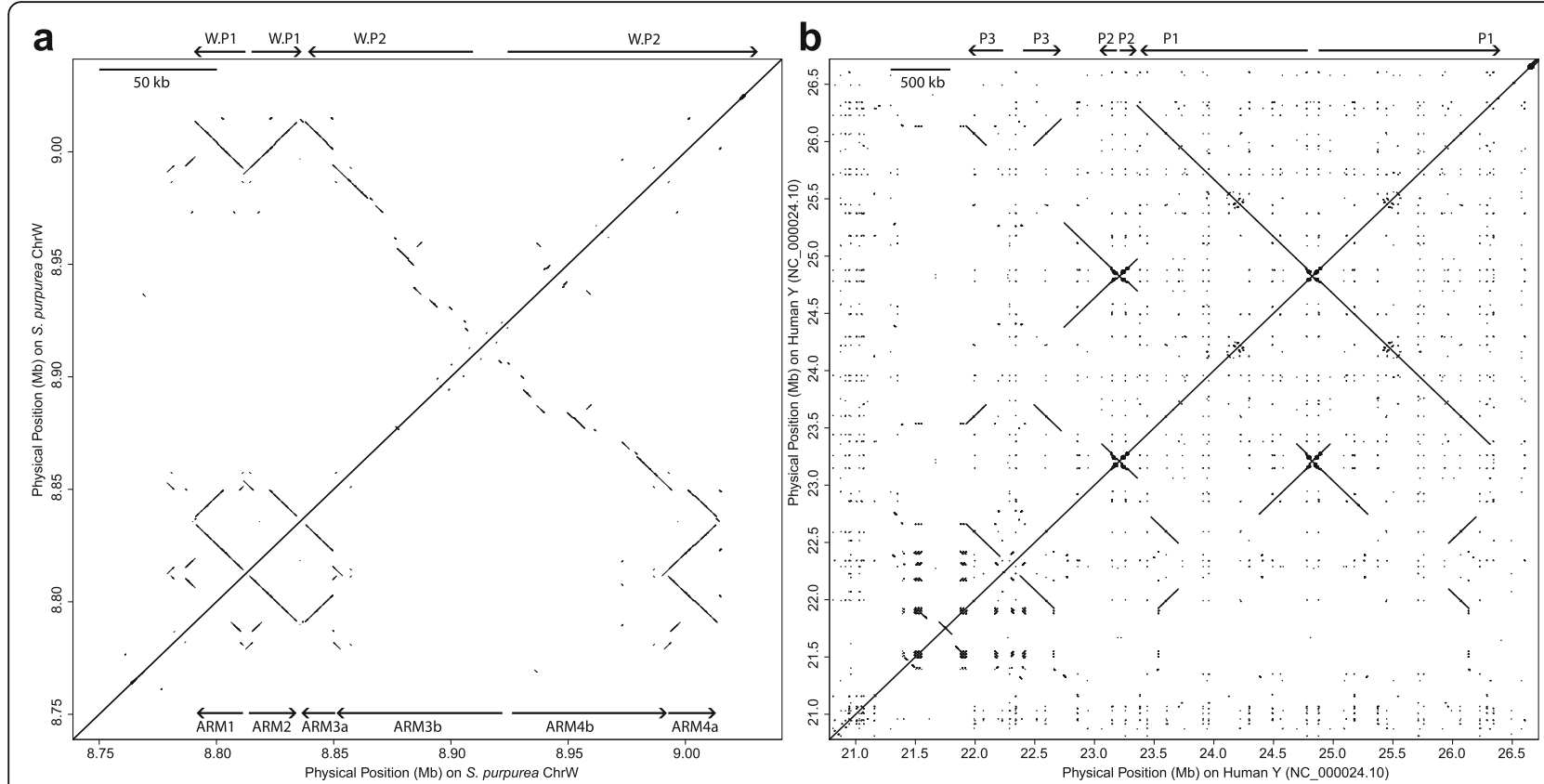

Fig. 4 Palindromic repeats in the S. purpurea W chromosome (a) and the H. sapiens $Y$ chromosome (b). The dot plots were produced using LASTZ with identical settings. Note the different scales, indicated by the bar at the top right of each figure. H. sapiens palindromes are labeled following Skaletsky et al. [4]

with a 2.6-kb spacer in the center, and Palindrome W.P.2 is immediately adjacent and spans over $165 \mathrm{~kb}$ (Table 2; Fig. 4a). A 20-kb sequence occurs in inverted orientation and shows high sequence identity across the four arms of both palindromes (Table 2; Fig. 5a). In palindrome W.P1, these are referred to as arm1 and arm2, and in Palindrome W.P2, these are referred to as arm3a and arm4a (Table 2; Fig. 4a). Sequence identity among these four arms is greater than $99 \%$ on average. The regions of high sequence identity are disrupted by a $\sim 500$ bp insertion in the center of arm4. Furthermore, arm3 has a $6.9 \mathrm{~kb}$ deletion at $11.7 \mathrm{~kb}$, followed by a stretch of $1.6 \mathrm{~kb}$ that can be aligned to the other arms in the same orientation (Fig. 5a). Additionally, there is a $12-\mathrm{kb}$ stretch upstream of arm 1 that shows high identity to portions of arms 1 and 2. We call this the pre-arm for convenience (Table 2).

Palindrome W.P2 contains an additional inverted repeat that is missing from W.P1. We refer to this as arm3b and arm4b (Table 2; Fig. 4a). Sequence identity is somewhat lower between these two arms compared to the other four, ranging from 96 to $99 \%$ over most of their length. Furthermore, the regions of high identity are disrupted by numerous insertions and deletions (Fig. 5b).

\section{Gene content of the palindromes}

There are five genes duplicated across arms 1, 2, 3a, and 4a of both palindromes. These are the Small Muts-Related protein (SMR), a Type-A cytokinin response regulator

Table 2 Coordinates of palindromes in the female SDR

\begin{tabular}{llllll}
\hline & Name & Start (bp) & End (bp) & Size (bp) & Gene families \\
\hline \multirow{2}{*}{ Palindrome W.P1 } & Pre-arm & $8,778,973$ & $8,791,042$ & 12,070 & R2,HCT \\
& arm1 & $8,790,932$ & $8,811,002$ & 20,071 & SMR, RR,R1,R2,HCT \\
& Spacer1 & $8,811,003$ & $8,814,588$ & 3586 & SMR,RR,R1,R2,HCT \\
Palindrome W.P2 & arm2 & $8,814,589$ & $8,834,138$ & 19,550 & SMR, RR, R1,HCT \\
& arm3a & $8,836,813$ & $8,850,772$ & 13,960 & DRBM,TF2C,DPRIM,DUF789 \\
& arm3b & $8,850,773$ & $8,920,527$ & 69,755 & DRBM,ACDP,DPRIM,DUF789 \\
& Spacer2 & Unidentified & & & SMR,RR,R1,R2,HCT \\
\hline
\end{tabular}




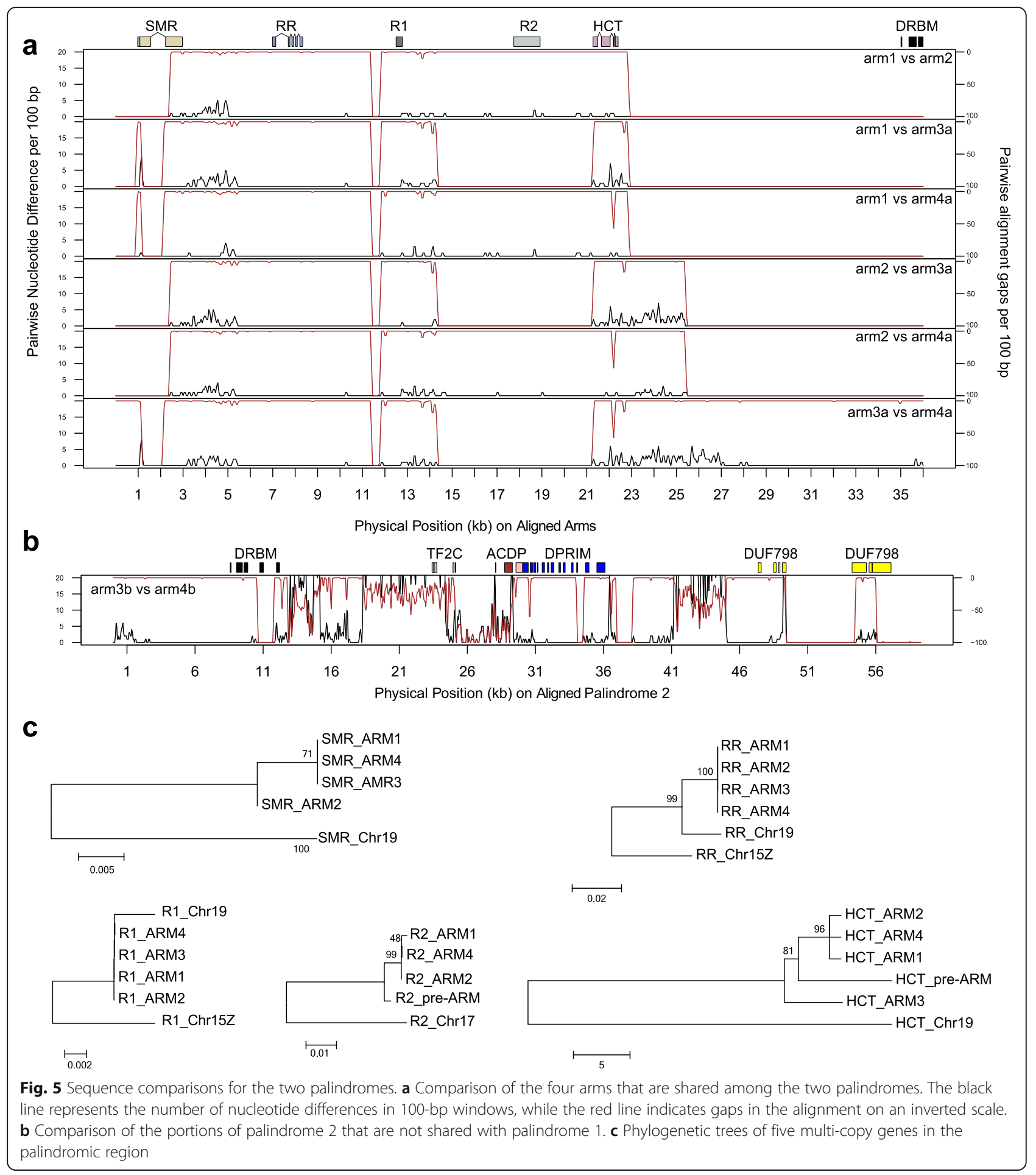

(RR), two genes that contain an NB-ARC domain (R1 and R2), and a hydroxycinnamoyl-CoA shikimate/hydroxycinnamoyl transferase (HCT) (Table 3). All of these genes except R2 have clear paralogous copies on Chr19. There is very little sequence divergence among most of these paralogs in the palindromes (Fig. 5).
The cytokinin response regulator is of particular interest because an ortholog of this gene has also been found to be associated with sex in Populus [24] and is therefore an excellent candidate as a sex determination gene in the Salicaceae. The RR gene is highly conserved across all four palindrome arms on the W-SDR (Fig. 5a, 
Table 3 Genes present in palindromes 1 and 2

\begin{tabular}{|c|c|c|c|c|c|c|c|c|}
\hline & $\begin{array}{l}\text { Gene } \\
\text { symbol }\end{array}$ & $\begin{array}{l}\text { Number } \\
\text { of copies }\end{array}$ & GenelD & $\begin{array}{l}\text { Chromosome } \\
\text { of the non-W } \\
\text { best hit }\end{array}$ & $\begin{array}{l}\text { Best hit in } \\
\text { A. thaliana }\end{array}$ & $\begin{array}{l}\text { Arabidopsis name or } \\
\text { description (function) }\end{array}$ & $\begin{array}{l}\text { Best hit in } P \text {. } \\
\text { trichocarpa } \mathrm{v} 3\end{array}$ & $\begin{array}{l}\text { Identity } \\
\text { of } P \text {. } \\
\text { trichocarpa } \\
\text { best hit }\end{array}$ \\
\hline \multirow{13}{*}{$\begin{array}{l}\text { Palindromes } \\
\text { W.P1 and } \\
\text { W.P2 }\end{array}$} & $S M R$ & $4^{[a]}$ & Manually annotated & Chr19 & AT5G23520 & $\begin{array}{l}\text { SMR (Small MutS Related) } \\
\text { domain-containing protein) }\end{array}$ & Potri.T013000 & 90.70 \\
\hline & \multirow[t]{4}{*}{$R R$} & \multirow[t]{4}{*}{4} & Sapur.15WG073500 & \multirow[t]{4}{*}{ Chr19 } & \multirow[t]{4}{*}{ AT3G56380 } & \multirow{4}{*}{$\begin{array}{l}\text { ARR17 (type A cytokinin } \\
\text { response regulator) }\end{array}$} & \multirow[t]{4}{*}{ Potri.019G133600 } & \multirow[t]{4}{*}{92.81} \\
\hline & & & Sapur.15WG073900 & & & & & \\
\hline & & & Sapur.15WG074000 & & & & & \\
\hline & & & Sapur.15WG075200 & & & & & \\
\hline & \multirow[t]{2}{*}{ R1 } & \multirow[t]{2}{*}{$4^{[a]}$} & Sapur.15WG073800 & \multirow[t]{2}{*}{ Chr15Z } & \multirow[t]{2}{*}{ AT4G27220 } & \multirow{2}{*}{$\begin{array}{l}\text { NB-ARC domain-containing } \\
\text { disease resistance protein }\end{array}$} & \multirow[t]{2}{*}{ Potri.T012900 } & \multirow[t]{2}{*}{81.00} \\
\hline & & & Sapur.15WG074100 & & & & & \\
\hline & $R 2$ & $3(1)^{[a]}$ & Manually annotated & Chr17 & AT4G27220 & $\begin{array}{l}\text { NB-ARC domain-containing } \\
\text { disease resistance protein }\end{array}$ & Potri.T013300 & 61.23 \\
\hline & \multirow[t]{5}{*}{ HCT } & \multirow[t]{5}{*}{$4(1)$} & Sapur.15WG073400 & \multirow[t]{5}{*}{ Chr19 $9^{[b]}$} & \multirow[t]{5}{*}{ AT5G48930 } & \multirow{5}{*}{$\begin{array}{l}\text { HCT (hydroxycinnamoyl-coa } \\
\text { shikimate/quinate } \\
\text { hydroxycinnamoyl transferase) }\end{array}$} & \multirow[t]{5}{*}{ Potri.018G104700 } & \multirow[t]{5}{*}{58.02} \\
\hline & & & Sapur.15WG073600 & & & & & \\
\hline & & & Sapur.15WG073700 & & & & & \\
\hline & & & Sapur.15WG074200 & & & & & \\
\hline & & & Sapur.15WG075100 & & & & & \\
\hline \multirow{8}{*}{$\begin{array}{l}\text { Palindrome } \\
\text { W.P2 only }\end{array}$} & \multirow[t]{2}{*}{$D R B M$} & \multirow[t]{2}{*}{2} & Sapur.15WG074300 & \multirow[t]{2}{*}{ Chr17 } & \multirow[t]{2}{*}{ AT1G09700 } & \multirow{2}{*}{$\begin{array}{l}\text { ATDRB1 (dsRNA binding } \\
\text { protein) }\end{array}$} & \multirow[t]{2}{*}{ Potri.017G126700 } & \multirow[t]{2}{*}{61.95} \\
\hline & & & Sapur.15WG075000 & & & & & \\
\hline & TF2C & 1 & Sapur.15WG074400 & Chr08 ${ }^{[c]}$ & AT2G27040 & $\begin{array}{l}\text { AGO4 (ARGONAUTE 4, siRNA } \\
\text { mediated gene silencing) }\end{array}$ & NA & NA \\
\hline & $A C D P$ & 1 & Sapur.15WG074900 & Chr17 & AT5G52790 & $\begin{array}{l}\text { CBS domain protein with } \\
\text { DUF21 (transmembrane } \\
\text { transporter) }\end{array}$ & Potri.017G147900 & 83.33 \\
\hline & \multirow[t]{2}{*}{ DPRIM } & \multirow[t]{2}{*}{2} & Sapur.15WG074500 & \multirow[t]{2}{*}{ Chr17 } & \multirow[t]{2}{*}{ AT5G52800 } & DNA primase & Potri.017G148000 & 92.52 \\
\hline & & & Sapur.15WG074800 & & & & & \\
\hline & DUF789 & 2 & Sapur.15WG074600 & Chr17 & AT1G03610 & DUF789 (protein of unknown & Potri.017G152600 & 86.03 \\
\hline & & & Sapur.15WG074700 & & & & & \\
\hline
\end{tabular}

${ }^{[a]}$ Manually annotated transcripts were included in the count. Numbers in the parentheses are from a fragment in the upstream portion of W.P1 that is homologous to part of W.P1. ${ }^{[b]}$ This cluster of tandem duplications on Chr19 in S. purpurea is not present on Chr19 in P. trichocarpa. ${ }^{[c]}$ The palindrome gene contains only a truncated blast hit to Sapur.008G005800 on Chr08. ${ }^{[\mathrm{d}]}$ This best hit with an expected value of $8 \times 10^{-3}$ due to a sequence length of 84 aa. Expected values of the remaining $A$. thaliana were less than $1 \times 10^{-10}$

c). Interestingly, we also found a pseudogene copy of the RR gene on the Z-SDR. This is the only one of the five genes that is present in some form on the W-SDR, the Z-SDR, Chr19, and also in the SDR of Populus. There is a 2.6-kb sequence inserted upstream of all $R R$ copies in the palindrome, and not in the Z-SDR pseudogene or on Chr19 (Additional file 2: Figure S5). This suggests that the W-SDR palindrome formed after transposition from Chr19. Interestingly, the RR gene also occurs as inverted repeats in all three locations in the genome (W-SDR, Z-SDR, and Chr19). However, alignment of the W-SDR, Z-SDR, and Chr19 versions demonstrates that the palindromes likely formed independently, because the palindromic regions are different (Additional file 2: Figure S5).

There are an additional five genes in the W.P2 palindrome. Three of these genes occur as inverted repeats: a
DNA-directed primase/polymerase protein $(D R B M)$, a DNA primase (DPRIM), and a protein containing Domain of Unknown Function 789 (DUF789). In addition, there is a homolog of ARGONAUTE 4 (TF2C) and a CBS domain protein $(A C D P)$ in single copy. Four of these genes were apparently transposed from Chr17 (Table 3). This leads us to the hypothesis that after these genes were transposed to the W-SDR they underwent several rounds of structural rearrangements, including duplications, inversions, and deletions.

\section{Multiple LTR retrotransposons in the palindrome}

To gain further insight into the composition and history of the W-SDR, we used LTRharvest and LTRdigest to annotate LTR retrotransposons in the palindromic region. We identified one LTR retrotransposon in the preArm region and 12 LTR retrotransposons in palindrome 
W.P2 that have terminal repeats identified with coding regions (Fig. 6a). These 13 retrotransposons are likely to be independent insertion events given that they have different long terminal repeats as well as different target site duplications and do not occur in the same position in the opposite arm of the palindrome (Additional file 1: Table S10). Given that there are varying numbers of substitutions within the LTRs of the same retrotransposon, it appears that these insertions have occurred repeatedly after establishment of the palindromes. Using a previous estimation of the mutation rate in $P$. tremula $\left(2.5 \times 10^{-9}\right.$ per year) [29], we estimate that the oldest insertion occurred at least $8.6 \pm 2.9$ s.d. MYA from a nonautonomous LTR retrotransposon, Ltr-p2-a (Fig. 6a and Additional file 1: Table S10). This is likely an underestimate, since the Salix substitution rate is substantially higher than that of Populus [30]. Since the oldest substitutions occurred in Palindrome W.P2, we infer that this element became established first (Fig. 6a). The LTRs of the nonautonomous elements Ltr-p2-a and Ltr-p2-k flank the $S M R$ and $R R$ genes (Fig. 6c, d; Additional file 2: Figure S6), which raises the intriguing possibility that these LTRs were involved in the transposition of these genes to this region. However, the target site duplications for these copies are identical across the palindrome arms, suggesting that the duplications and rearrangements of these genes in the W-SDR did not involve these elements (Additional file 2: Figure S6). We also found two highly similar LTRs from the same family in W.P1 (Ltr-p2-b3 on arm3 and the Ltr-p2-b4 on arm4; Fig. 6a-c; Additional file 1: Table S10). There are truncated parts of this LTR in the pre-arm and the spacer

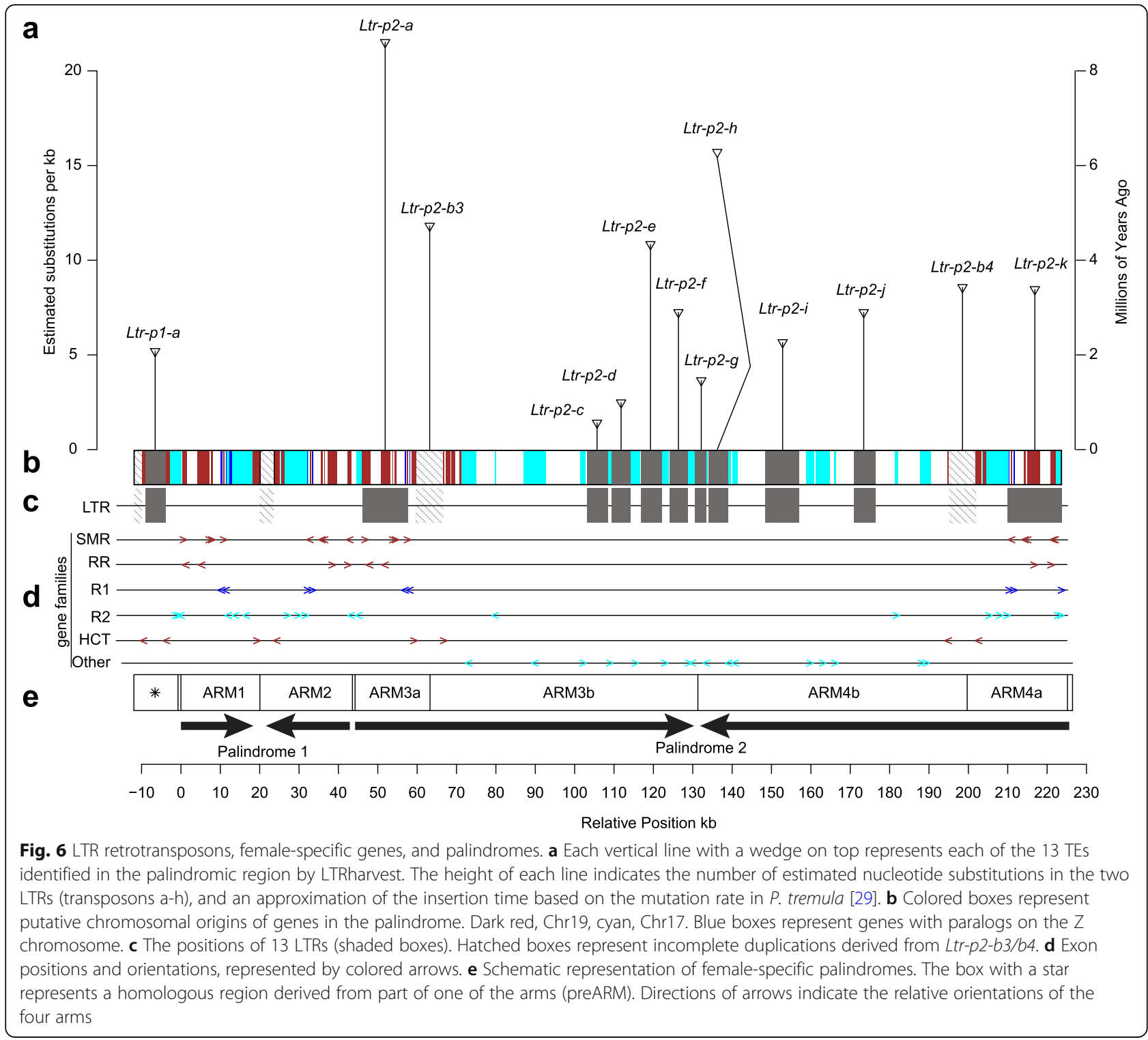


between arm 1 and arm 2 as well (Fig. 6b, c). These copies might be a direct consequence of duplications and inversions that occurred during the formation of the palindrome instead of independent insertions.

\section{Evidence for gene conversion in the palindromes}

We have shown that the palindromes are likely to be millions of years old based on the retrotransposon analysis, yet sequence identity of portions of the palindrome arms remains high (Fig. 5a). The most parsimonious explanation for this is gene conversion among the palindrome arms, as has been observed in the mammalian Y chromosome palindromes $[6,31]$. To test for this, we searched for regions that had interspecific base substitutions relative to Salix suchowensis, a closely related species with ZW sex determination [22]. If regions with interspecific substitutions lack paralogous sequence variation (PSV) across the palindrome arms, then this would be excellent evidence of gene conversion [31]. We detected a 3-kb region within the palindromes where there are no PSVs in S. purpurea and only one PSV in $S$. suchowensis, but substantial interspecific polymorphisms (Fig. 7). The depth of this region is $4 \mathrm{~N}$ as expected for the four copies of the palindrome arms in S. purpurea. In $S$. suchowensis, the depth is between $2 \mathrm{~N}$ and $3 \mathrm{~N}$, which indicates that there might be a palindrome structure as well, though it might be incomplete. We also applied the same methods with resequencing reads of two female and two male $S$. viminalis individuals (another Salix with ZW sex determination) [21], but the palindromic region was not well covered by reads of either sex. This may indicate that $S$. viminalis lacks the palindrome, though it is more distantly related to $S$. purpurea than is $S$. suchowensis, so this may simply be due to excessive sequence divergence in this region.

\section{Expression patterns of genes in the palindromes}

We examined expression profiles in multiple tissues of the two reference genomes to validate the predicted transcripts and to determine how the expression patterns of genes in the palindromes differ from their autosomal counterparts. Most genes in the palindromes show female-limited expression while the autosomal copies are generally not sex-biased (Fig. 8a). The cytokinin response regulator $(R R)$ (Sapur.15 W073500) shows the highest expression in catkin tissue, followed by expression in shoot tips and stems. On the contrary, two autosomal copies on Chr19 show lower expression, limited to female catkins and male buds. The four copies of the SMR gene show low expression in female catkins and other tissues, but the autosomal copy on Chr19 (Sapur.019G001500) is expressed in all tissues (Fig. 8a). All five copies of the $H C T$ gene from the palindromes showed low expression in female catkins and roots and higher expression in leaf tissues, shoot tips, and stems, all of which were female-biased. Two copies of the DNA

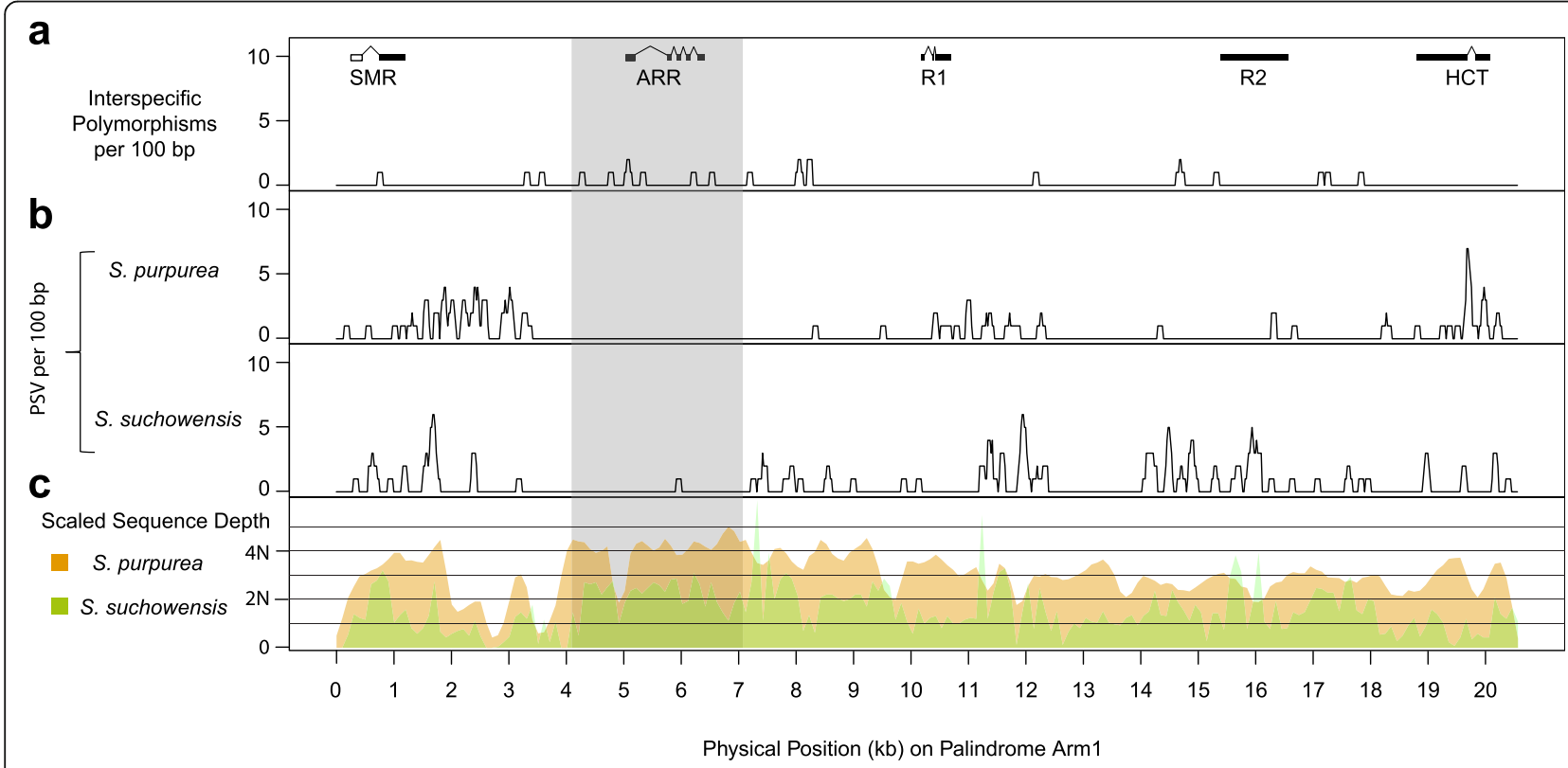

Fig. 7 Sequence variation in the palindrome arms. a Density of fixed differences between S. purpurea and S. suchowensis per $100 \mathrm{bp}$. b Density of paralogous sequence variants (PSVs, differences among the four palindrome arms) in S. purpurea and S. suchowensis. c Relative depth of Illumina sequence reads aligned to a reference sequence of one arm of the S. purpurea palindrome, where $2 \mathrm{~N}$ represents the expected depth of read alignment across the whole genome. The gray shaded area represents a segment of the palindrome that is enriched for interspecific fixed variants, but depleted in PSVs, providing strong evidence for differential gene conversion in the two lineages 


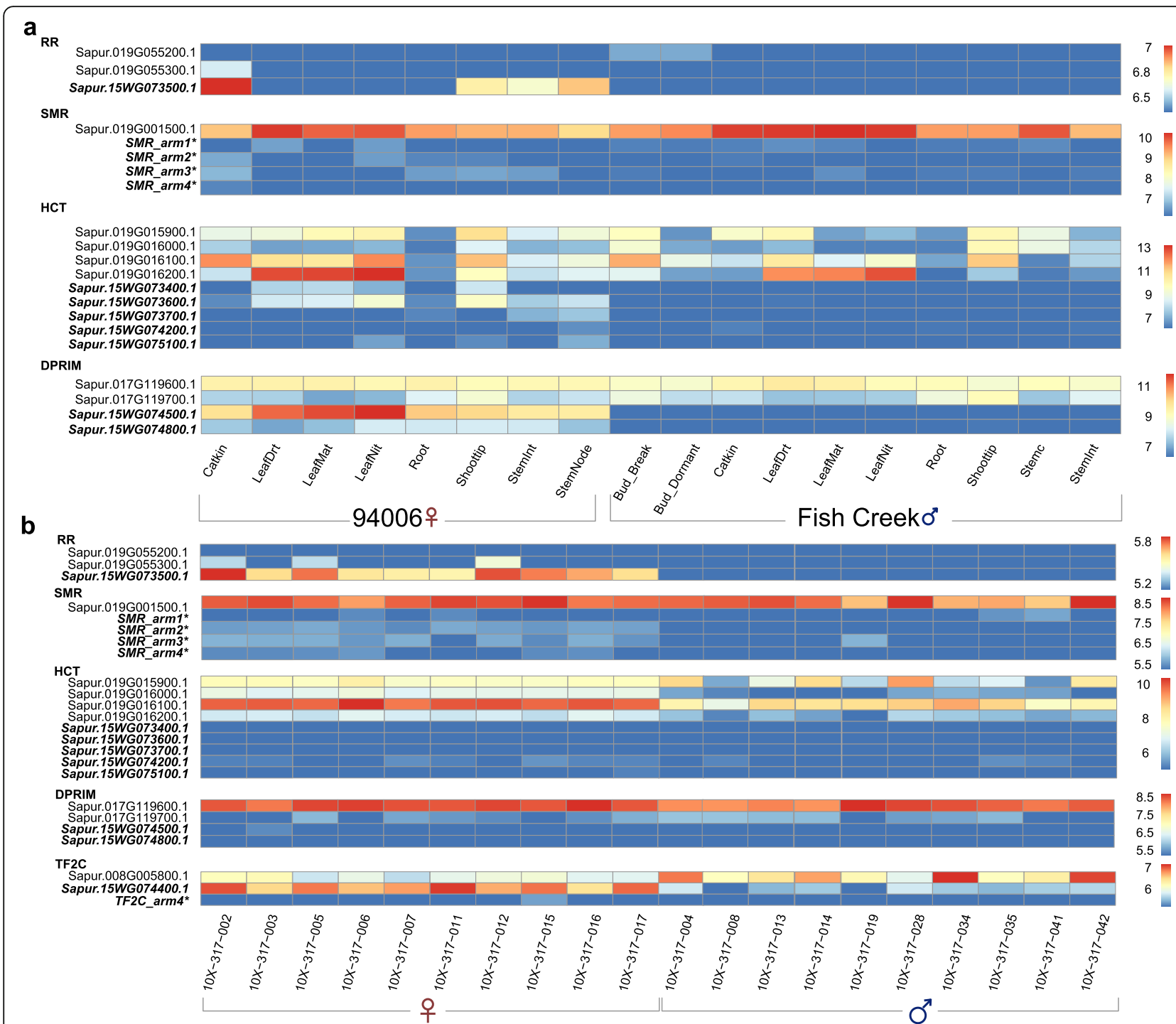

Fig. 8 Expression profile of genes from the W palindromes and autosomal paralogs. a Normalized read counts of genes in different tissues from clone 94006 (female) and Fish Creek (male). b Normalized read counts of selected genes in catkins from 10 females and 10 males from an $F_{2}$ family. Gene labels in bold font are from the palindromes. Asterisks indicate manually annotated genes

Primase gene from palindrome W.P2 also show high expression in leaf tissues while the original copy on the autosome (Sapur.017G119600) was expressed across all sampled tissues. Similarly, analysis of transcriptomic data of catkins from 10 females and 10 males in the $F_{2}$ family confirms that the genes in the palindromes are primarily expressed in female tissue, in contrast to their autosomal paralogs (Fig. 8b).

\section{Discussion}

\section{The W chromosome in S. purpurea}

Using depth of coverage for males and females from a controlled cross pedigree, we have been able to identify $\mathrm{Z}$ and W haplotypes from the SDR of a highly heterozygous species from a standard PacBio assembly. We also show how presence-absence markers generated from sequence depth in controlled cross progeny can be used to genetically map hemizygous portions of the SDR. In a similar study of a young $\mathrm{Y}$ chromosome in asparagus, BioNano optical maps for a YY individual were generated to improve genome contiguity, and sequence depth of coverage was also treated as a QTL to aid the assembly because of the presence of large indels in the sex chromosome [16]. Here, we showed that by combining long-read sequencing with GBS marker data from a large $\mathrm{F}_{2}$ family, we could efficiently identify the male and female haplotypes in the SDR. However, unlike strategies like single-haplotype iterative mapping and sequencing (SHIMS) that have been used in assemblies of mammalian Y chromosomes [4, 32-34], our map-based strategy 
could not provide a definitive order for the W contigs due to lack of recombination in the SDR.

The W-SDR is approximately $2.5 \mathrm{Mb}$ larger than the ZSDR. This is due in part to a greater accumulation of transposable elements, which account for approximately $1.35 \mathrm{Mb}$ of this difference. This is consistent with expectations for sex chromosome evolution where transposable elements are expected to accumulate in regions with suppressed recombination $[1,35,36]$. However, gene content of the sex chromosome is expected to decrease due to the absence of recombination and reduced efficiency of purifying selection $[1,27]$. Instead, we observed that gene content is expanded in the W-SDR, driven in part by numerous transpositions and subsequent expansion of autosomal genes. Autosomal transpositions have also been demonstrated in other sex chromosomes, including mammalian Y chromosomes [6]. The recently formed neo-Y chromosome of Drosophila miranda also shows massive expansion of genes that have been translocated from autosomes, and these are enriched for genes contributing to sex-specific functions [37].

Sex chromosomes commonly show evidence of "evolutionary strata" with markedly different levels of sequence divergence that represent different epochs of expansion of the SDR [35]. Under one common model of sex chromosome evolution, these strata are the result of multiple periods of SDR expansion as sexually antagonistic polymorphisms become incorporated into the SDR $[27,38]$. Although the identified SDR in S. purpurea is about 6-7 Mb, occupying more than one third of the $\mathrm{W}$ chromosome assembly, we detected little evidence for the existence of such strata. This corroborates a previous analysis that failed to detect strata in S. suchowensis using an integrated segmentation and clustering method [39]. It appears that cessation of recombination has not been a gradual long-term process in the $S$. purpurea SDR, although it is certainly possible that the oldest strata have decayed to the point where they cannot be meaningfully aligned. An explanation for the large size of this region is that it partially overlaps with the centromere of Chr15, as we previously reported [20]. It is possible that the repressed recombination in this region pre-dated the transposition of a relatively small SDR cassette, as has been observed in octoploid Fragaria [17]. This is consistent with the apparently small size of the region in Populus $(\sim 100 \mathrm{~kb})$, which is located on a different chromosome [24]. This is also consistent with the structure and composition of the palindromic repeats that we discovered in S. purpurea, which are excellent candidates as sex determination loci, as detailed below.

\section{Sex chromosome palindrome repeats}

We have reported here the first observation of a large inverted repeat in a plant sex chromosome, similar to the palindromic structures observed in mammalian sex chromosomes. We have further demonstrated that these palindromes are undergoing gene conversion, suggesting functional similarities to mammalian sex chromosome palindromes. W.P1 and W.P2 of S. purpurea have a similar arrangement of arms as P1 and P3 in humans due to the presence of highly homologous regions between the two palindromes. Similar palindromes have been also been discovered on Y chromosomes of other mammals, as well as avian $\mathrm{W}$ chromosomes (reviewed by $[5,6])$. Large mammalian palindromes developed as a series of accumulations of insertions from autosomes and maintained through arm-to-arm gene conversion. This intrachromosomal gene conversion can maintain coding sequence integrity which otherwise would be compromised by the continuous accumulation of deleterious mutations in the absence of homologous recombination (i.e., Muller's ratchet) $[5,6,31,40]$. The fact that these structures have independently evolved in nonrecombining regions of sex chromosomes is an intriguing case of convergent evolution of chromosome structure. Interestingly, the chloroplast genome, another non-recombining chromosome in plants, also contains a different large inverted repeat that undergoes gene conversion [41] and helps maintain structural integrity of the genome, suggesting that this phenomenon may be common in regions of the genome that lack recombination [42]. However, it is also important to note that not all palindromic repeats occur in regions of the genome with suppressed recombination, most notably the large palindromes on the mammalian $\mathrm{X}$ chromosome. Palindromes may therefore play another role beyond maintenance of sequence integrity, such as mitigating expression of sexually antagonistic genes [9] or in gene dosage compensation in the heterogametic sex [43, 44].

The S. purpurea palindromes are considerably smaller than mammalian palindromes and have only accumulated two major autosomal transpositions (from Chr17 and Chr19), possibly reflecting their young age. Another difference between the human palindrome and the one in S. purpurea is that the gene conversion seems to be quite efficient across all the eight palindromes in humans, but the observed regions under gene conversion in S. purpurea are much more limited. This is particularly obvious in W.P2, compared to human P1, which has high sequence identity over several megabases (Fig. 4). Nevertheless, we found strong evidence for gene conversion in the cytokinin response regulator gene, based on an absence of PSVs. The ortholog of this gene in S. suchowensis has accumulated divergent nucleotide substitutions, which also seem to be homogenized among copies. This is a clear signature of gene conversion and is unlikely to result from purifying selection or very recent independent duplication events [31]. 


\section{Evidence for a possible shared evolutionary history for the Populus and Salix SDRs}

Initial analyses in P. trichocarpa suggested that the SDR is much younger than the whole genome duplication event that is shared by Populus and Salix, suggesting that the SDR became established well after these genera diverged [24]. The low divergence between homologs in the fully sex-linked region (i.e., between Chr15W and Chr15Z homologs) shows that the SDR of S. purpurea evolved recently. Furthermore, given that the SDR is located in approximately the same portion of Chr15 in both S. purpurea and S. suchowensis, and both have ZW systems [20, 22], it is reasonable to assume that the SDR became established in this lineage prior to divergence of these two species, but well after divergence from Populus, which has an XY SDR on Chr19. On this basis, it has been hypothesized that these SDRs have independent evolutionary origins [22]. We believe that our results point toward a single origin of dioecy in these genera, as well as shared components of an underlying sex determination system focused on cytokinin-mediated regulation.

Support for this hypothesis is provided by the type A cytokinin response regulator homologs that occur in palindrome arms 1,2,3a, and 4a (Table 3), which show strong evidence of ongoing gene conversion and femalespecific expression in S. purpurea. The best ortholog of these genes in P. trichocarpa is Potri.019G133600 (this gene was originally designated PtRR11, but it is referred to as $R R 9$ in subsequent publications [45-47], so we will adopt that nomenclature here to avoid confusion). PtRR9 grouped with the Arabidopsis thaliana type A response regulators ARR16 and ARR17 in the original phylogenetic analysis of this family in Populus [48]. The ARR16 gene has been implicated in gynoecial development in Arabidopsis [49]. PtRR9 is expressed primarily in reproductive tissues in Populus $[47,48]$ and is also associated with sex in several Populus species [24, 45, 46]. Further supporting its possible role in sex determination, it was the only gene in the P. balsamifera genome that showed clear sex-specific differences in promoter and gene body methylation [45]. This raises the intriguing possibility the mechanisms of sex determination in ZW Salix and XY Populus share common regulatory elements and a shared evolutionary origin.

The cytokinin signaling pathway has emerged in recent years as a prominent candidate for regulating floral development and sex expression in plants $[50,51]$. The potential role of cytokinin signaling in dioecy has recently been highlighted by the groundbreaking study by Akagi et al. in kiwifruit (Actinidia spp.) [51]. The authors identified a Type C response regulator (Shy Girl, SyGI) on the $\mathrm{Y}$ chromosome that was associated with maleness. Overexpression of this gene in Arabidopsis and
Nicotiana tabacum caused suppression of carpel development, supporting its potential role as a suppressor of female function [13]. This work has some interesting parallels with the results reported here for Salix and Populus. First, type $\mathrm{C}$ response regulators are essentially similar in structure to Type A response regulators, with the main difference being that Type $\mathrm{C}$ is not induced by cytokinin. Interestingly, PtRR 9 also was not induced by exogenous cytokinin application [48], though this has not yet been tested with floral tissue. Second, SyGI was duplicated from an autosomal gene and subsequently gained a new function on the $\mathrm{Y}$ chromosome, much like SpRR9 has been duplicated from Chr19 in S. purpurea and established a distinct pattern of expression, and presumably new functions. However, RR9 and SyGI are clearly not orthologous and likely perform different roles in cytokinin signal transduction. This supports the view that there are numerous ways to achieve separate sexes in plants, and it is likely that a myriad of mechanisms underlie the hundreds of independent occurrences of dioecy in the angiosperms [52], even if a relatively small number of pathways are involved $[13,53]$.

\section{Conclusion}

We have shown that the SDR on the W chromosome of S. purpurea has expanded gene content compared to the corresponding region on the $\mathrm{Z}$ chromosome, due in part to autosomal genes that have been transposed and expanded in the region of suppressed recombination. We further demonstrated that some of these transposed genes are arranged as palindromic repeats that are undergoing gene conversion, suggesting some functional similarities to the mammalian sex chromosomes. This is a striking example of convergent evolution in chromosome structure. We have also demonstrated that the coding sequence undergoing gene conversion in the palindrome, $\operatorname{SpRR}$, is orthologous to a gene that is also associated with sex in Populus. This gene is an excellent candidate for controlling sex determination through modulation of the cytokinin signaling pathway. However, much remains to be determined about the underlying mechanism of sex determination. Most importantly, it is currently unclear how the same gene is functioning in an XY system in Populus and a ZW system in Salix. It is possible that the $\mathrm{W}$ chromosome version acts as a dominant promoter of female function, while the $\mathrm{Y}$ version is a dominant suppressor of female function, based on the putative roles of cytokinin and the type A response regulators in female development in Arabidopsis. A detailed model should emerge through comparative analysis of the $\mathrm{W}$ and $\mathrm{Y}$ chromosomes of multiple species in the Salicaceae, which is currently underway. If the underlying mechanism shares common regulatory elements, 
this will be the first case demonstrating $\mathrm{XY}$ and $\mathrm{ZW}$ systems that are controlled by the same pathway in plants.

\section{Methods}

\section{Initial assembly of the genome}

Whole genome assemblies were produced for two $S$. purpurea clones: female clone 94006, and a male offspring of this clone, "Fish Creek" (clone 9882-34), which was derived from a controlled cross between clone 94006 and male S. purpurea clone 94001. Clones 94001 and 94006 were collected from naturalized populations in upstate New York, USA. Sequencing reads were collected using the Illumina and PACBIO platforms at the Department of Energy (DOE) Joint Genome Institute (JGI) in Walnut Creek, California, and the HudsonAlpha Institute in Huntsville, Alabama. Illumina reads were sequenced using the Illumina HISeq platform, and the PACBIO reads were sequenced using the RS platform. One 400 bp insert $2 \times 250$ Illumina fragment library was sequenced for total coverage of $183 \times$ in clone 94006 and 153x in Fish Creek. Prior to use, Illumina reads were screened for mitochondria, chloroplast, and ФX174 contamination. Reads composed of $>95 \%$ simple sequence were removed. Illumina reads $<50 \mathrm{bp}$ after trimming for adapter and base quality $(q<20)$ were removed. For the PACBIO sequencing, a total of $47 \mathrm{P} 6 \mathrm{C} 4$ chips $(10 \mathrm{~h}$ movie time) were sequenced for each genome with a pread yield of $39 \mathrm{~Gb}$ and a total coverage of $\sim 110 \times$ per genome (Additional file 1: Table S11). The assembly was performed using FALCON-UNZIP [54], and the resulting sequence was polished using QUIVER [55]. Finally, to correct false polymorphisms resulting from errors in PacBio reads, homozygous SNPs and INDELs were corrected in the release consensus sequence using $\sim 80 \times$ of the $2 \times 250$ Illumina reads from the reference individual. This was accomplished by aligning the reads using bwa mem and identifying homozygous SNPs and INDELs with the GATK's UnifiedGenotyper tool [56] (Additional file 1: Table S12).

Chromosome-scale assemblies were created using a genetic map derived from 3697 GBS markers generated for a family of $497 F_{2}$ progeny from a cross in which the male reference is the father and the female reference is the grandmother. This map is described more completely in a previous publication [57]. This intercross map was used to identify misjoins, characterized by an abrupt change in the $S$. purpurea linkage group. Scaffolds were then oriented, ordered, joined, and numbered using the intercross map and the existing $94006 \mathrm{v} 1 \mathrm{re-}$ lease assembly [20]. Adjacent alternative haplotypes were identified on the joined contigs, and these regions were then collapsed using the longest common substring between the two haplotypes. Significant telomeric sequence was identified using the (TTTAGGG) $)_{n}$ repeat, and care was taken to make sure that it was properly oriented in the production assembly. The remaining scaffolds were screened against bacterial proteins, organelle sequences, and GenBank nr and removed if found to be a contaminant. Completeness of the euchromatic portion of the assembly was assessed by aligning S. purpurea var 94006 v1 annotated genes to the assemblies. In both cases, $99.7 \%$ of the genes were found.

\section{Identification of $\mathrm{W}$ contigs}

Contigs derived from the $\mathrm{W}$ chromosome are expected to contain some large indels compared to contigs from the $\mathrm{Z}$ chromosome due to the lack of recombination between $\mathrm{W}$ and $\mathrm{Z}$. These hemizygous regions should exclusively occur in the $\mathrm{W}$ haplotype of SDR. To identify these regions, we aligned $2 \times 250 \mathrm{bp}$ Illumina resequencing reads from female clone 94006 and male clone Fish Creek to the new reference using Bowtie2 [58]. Depth of coverage was extracted using samtools-1.2 [59]. Median depth was calculated using a non-overlapping sliding window of $10 \mathrm{~kb}$.

To verify if these hemizygous regions are strictly inherited in only female individuals, we used the GBS data from the $F_{2}$ family. GBS reads of 195 offspring of each sex were aligned to the v5 reference with Bowtie2. Due to low coverage and depth of the GBS markers per locus per individual, bam files were merged according to sex in samtools-1.2. Depth was then called in Samtools-1.2 with and max depth was limited to 80,000. Regions continuously covered by GBS reads were defined as GBS intervals. Then, the median of each sex was calculated across all of the intervals. We defined markers as female-specific by integrating the depth from both the $F_{2}$ GBS and $2 \times 250$ datasets (restricted to the GBS intervals) using two rules: (1) $\log _{2}\left(\frac{M_{195}+1}{F_{195}+1}\right)<L$, where $L$ is the lower bounds of the distribution, defined by the fifth percentile divided by the number of intervals tested (Additional file 2: Figure S7), and (2) $\log _{2}\left(\frac{94006_{2 b 2550}+1}{\text { Fish Creek } 2 b y 250+1}\right)>5$. The cutoff for the second criterion was based on the occurrence of a distinct peak in the distribution of the ratios (Additional file 2: Figure S8). Scaffolds that contained at least three sex-linked markers were selected as candidate $\mathrm{W}$ scaffolds. Based on these criteria, only two contigs from the original Chr15 assembly were from $\mathrm{W}$ contigs, and the rest were from $\mathrm{Z}$ (Additional file 1: Table S5; Additional file 2: Figure S1a).

\section{Assembly of the $\mathbf{Z}$ and $\mathbf{W}$ chromosomes}

Raw GBS reads used for the original map were demultiplexed and trimmed down to $64 \mathrm{bp}$ for each read by process_radtags (in Stacks 1.44 [60]) with -c -q -r -t 64. Then, trimmed reads of each sequenced individual from the $F_{2}$ family were aligned to the 19 chromosomes and unmapped scaffolds from the main genome and 
alternative haplotypes from the v4 reference of 94006 using Bowtie 2 [58] with the --very-sensitive flag (-D 20 $-\mathrm{R} \quad 3 \quad-\mathrm{N} \quad 0 \quad-\mathrm{L} \quad 20$-i $\mathrm{S}, 1,0.50)$ to maintain a balance between sensitivity and accuracy. Upon examining the distribution of SNPs in the genome, it became clear that the alternative haplotypes were preventing us from retrieving markers in some regions in the genome, so we repeated the alignments using three different reference sequences: (1) the 19 chromosomes, (2) unmapped scaffolds, and (3) alternative haplotypes. Then, a wrapper script ref_map.pl in Stacks was used to call genotypes with $-\mathrm{m} 5$ (minimum number of reads to create a tag for parents) and -P 3 (minimum number of reads to create a tag for an offspring) on all progeny. Cross type "CP" was chosen since it was the one closest to our cross. Offspring with poor coverage were removed from the downstream analysis.

Once all genotypes were retrieved through Stacks, markers from different loci showing the exact same genotype/segregation across the progeny were binned and only markers from the main genome were kept for mapping. Markers with severe segregation distortion or excessive missing data were excluded, along with 12 offspring with a very low call rate. Genotypes were imputed and corrected based on inferring haplotypes in the two $\mathrm{F}_{1}$ parents from segregation of the markers in the progeny.

The grandparents of the $F_{2}$ cross have extensive stretches of shared haplotypes, possibly due to historic inbreeding in this naturalized population. This results in long runs of heterozygosity and homozygosity in the $F_{1}$ progeny. This inhibits integration of backcross and intercross markers by available mapping algorithms like those in the Onemap package [61]. To circumvent this problem, all intercross markers were translated to female and male backcross markers by identifying the parental origins of alleles based on parental phases and physical position in the assembly. Also, putatively hemizygous markers were recoded as backcross markers using sequence depth to infer genotypes. For example, markers with the segregation pattern $+/-\mathrm{x}-/$ - were recoded as $\mathrm{AB} \times \mathrm{BB}$. These genotypes were also imputed and corrected based on the inferred haplotypes of the two F1 parents.

Onemap v2.1.1 was used to form initial linkage groups. For each chromosome, there are two phased linkage groups from each backcross type. However, this phase information derived from the $F_{2}$ family is only for the $F_{1}$ parents, which cannot be directly used for phasing haplotypes in the grandmother, clone 94006 . By comparing parental genotypes from one LG to those of the grandparents, we inferred which of the 94006 haplotypes were inherited by each $F_{1}$. These results were used as a piece of evidence for identifying W-linked scaffolds/contigs, as well as estimating the overall occurrence of chimeric contigs in the assembly. After building a framework genetic map using markers from the main genome, nondistorted markers from unmapped main scaffolds and alternative scaffolds were added.

All unmapped scaffolds were manually checked to see if they matched the phase information or contained sexlinked markers. Those that were identified as $\mathrm{Z}$ scaffolds/contigs were excluded from the $W$ map. The new $\mathrm{W}$ and $\mathrm{Z}$ were assembled using the python package ALLMAPS [62] to order and orient scaffolds and reconstruct chromosomes based on the genetic map. Only the order of the female backcross map was used to assemble the W, and ALLMAPS was set not to break contigs. This new map-based assembly containing two versions of chromosome 15 (Chr15Z and Chr15W) is version 5 of the $S$. purpurea var 94006 genome.

To identify Z-W homologous regions (analogous to Xdegenerate regions in mammalian sex chromosomes) and insertions in the $\mathrm{W}$ haplotype, we realigned the $2 \times$ 250 reads of 94006 and Fish Creek to the 94006 v5 reference using Bowtie 2 as described above, except we removed Chr15Z from the reference. Depth was calculated using samtools, and the median depth of 50-kb nonoverlapping windows was calculated with an in-house perl script. Regions where medians of Fish Creek depth are no greater than 10 were considered as insertions in the FSW, and regions with greater depth were considered Z-W homologous regions. This analysis was repeated with a 10-kb window as well to enhance the resolution.

\section{Annotation of the genome}

Transcript assemblies were constructed from $\sim 126 \mathrm{M}$ pairs of $2 \times 76$ bp (94006) or $2 \times 150$ bp (Fish Creek) paired-end Illumina RNA-seq reads using PERTRAN. A total of 188,628 transcript assemblies were constructed using PASA from the RNA-seq transcript assemblies. Loci were determined by transcript assembly alignments and/or EXONERATE alignments of proteins from Arabidopsis thaliana, soybean, poplar, cassava, brachypodium, grape, and Swiss-Prot proteomes, and high confidence Salix purpurea Fish Creek gene model peptides, with up to $2 \mathrm{~kb}$ extension on both ends unless extending into another locus on the same strand. The reference genome was soft-masked using RepeatMasker. Gene models were predicted by the homology-based predictors. FGENESH+, FGENESH_EST, and EXONERATE, by PASA assembly of ORFs, and from AUGUSTUS via BRAKER1. The best scored predictions for each locus were selected using multiple positive factors including EST and protein support, and one negative factor: overlap with repeats. The selected gene predictions were improved by PASA. Improvement included adding UTRs, 
splicing correction, and adding alternative transcripts. PASA-improved gene model proteins were subjected to protein homology analysis to the abovementioned proteomes to obtain Cscore (the ratio of mutual best hit BLASTP scores) and percentage of protein aligned to the best homolog. The transcripts were selected if its Cscore was greater than or equal to 0.5 and protein coverage greater than or equal to 0.5. Alternatively, proteins with EST coverage were accepted if overlap with repeats was less than $20 \%$. For gene models with greater than 20\% CDS overlap with repeats, the Cscore cutoff was 0.9 and homology coverage was at least $70 \%$. The selected gene models were subjected to Pfam analysis, and gene models with more than $30 \%$ in Pfam TE domains were removed. Incomplete gene models with low homology and transcriptome support and short single exon proteins $(<300$ BP CDS) lacking conserved domains or transcriptome support were manually filtered out.

To annotate potential genes or coding regions in the palindrome that were missed by the automated annotation, the full nucleotide sequence of arm 1 (about $20 \mathrm{~kb}$ ) was submitted to the Fgenesh online service (http://www. softberry.com/berry.phtml?topic=fgenesh) with specific gene-finding parameters for Populus trichocarpa. The predicted peptide sequences were searched against predicted proteins from Populus trichocarpa v3.0, and Arabidopsis thaliana TAIR10 in Phytozome 12 (https://phytozome.jgi. doe.gov/) to find the closest homologous annotation. The protein domains were identified using hmmscan in HMMER (v3.1b1, http://hmmer.org/) against the Pfam-A domains (release 32, https://pfam.xfam.org).

\section{Comparison of $\mathrm{Z}$ and $\mathrm{W}$ orthologous genes}

Homologous genes on the $\mathrm{Z}$ and $\mathrm{W}$ chromosomes (Z-W homologs) were identified by performing a reciprocal blastp of all primary annotated peptide sequences in the main genome with default parameters. Mutual best hits were identified with over $90 \%$ identity over at least $70 \%$ of the transcript. Tandem duplications were identified as genes with expectation values of $1 \times 10^{-10}$ that occurred within a 500-kb window. In these cases, one representative gene from each tandem array was used as a representative sequence, and the mutual best hit outside the tandem array was identified as above. Genes that lacked hits in the Z-SDR were searched against the Populus trichocarpa v3.0 reference genome. Those with hits to Chr15 in Populus were designated as "Ancestral" under the assumption that the homolog was present prior to the establishment of the SDR in S. purpurea, but was subsequently lost from the Z-SDR. Those genes that lacked hits to Chr15 in either species but which had a mutual best hit meeting the above criteria to an autosomal gene were designated as autosomal transpositions.
Genes that could not be readily categorized due to a lack of mutual best hits satisfying the above criteria were designated as "Non-mutual" or "No Hit" as appropriate.

To identify homologous gene pairs for calculation of synonymous substitutions between the $\mathrm{Z}$ and $\mathrm{W}$ alleles, a reciprocal blast of all primary annotated peptide sequences was run with "blastall -p blastp -i -e 1e-20 -b 5 -v 5 - $\mathrm{m}$ 8", and MCscanX was run with default parameters [63]. The synonymous and nonsynonymous substitution rate of each gene pair in each syntenic block $\left(d_{S}\right.$ and $d_{\mathrm{N}}$, respectively) was estimated by aligning the sequences with CLUSTALW [64] and using the yn00 function in PAML [65]. Only pairs between the W-SDR and Z-SDR (including the unmapped scaffold_844) were used for estimating the divergence between $\mathrm{Z}$ and $\mathrm{W}$ haplotypes. It is important to note that this analysis does not control for polymorphism within populations, so it may be an overestimate of divergence.

\section{Identification of sex-associated loci}

Loci associated with sex were identified using 60 nonclonal individuals from a naturalized population of $S$. purpurea [66]. GBS reads from each individual were aligned to the 94006v5 genome without Chr15Z using Bowtie2. Genotypes were called in Stacks 1.14 using the ref_map.pl wrapper and the population module with a minimum minor allele frequency of 0.1 and a genotyping rate of 0.1 . Loci with greater than $40 \%$ missing data were removed. Association with sex was performed using emmax [67] as described previously [20].

\section{Detection of palindromic repeats}

We detected the palindromic repeats by aligning the SDR region to itself with LASTZ 1.03.66 with the following flags: --gapped --exact $=100$--step $=20$. Paralogous gene copies on autosomes were retrieved from the reciprocal blastp results described above. Paralogous genes within the palindrome arms were aligned along with paralogous copies from the autosomes using MUSCLE using default parameters provided in MEGA 5. In a few cases, the resulting alignments were adjusted manually (Supplemental Materials: Additional file 3). A NeighborJoining tree with default parameters was built using MEGA 5 [68].

To identify recent insertions of transposable elements within the palindrome, LTRharvest [69] was run with the sequence of the palindromic portion of the W-SDR from 8778 to $9015 \mathrm{~kb}$ with the target site duplication restricted to 5 to $20 \mathrm{bp}$. To find the protein domains in the coding region, a protein domain search against Pfam-A domains (release 32) was performed using the hidden Markov model methods implemented in LTRdigest (-hmms flag) [70]. Predicted LTR retrotransposons 
were determined to be non-automonous when coding regions did not contain any gag-or pol-related domains.

We estimated time since transposition based on the number of substitutions between the two LTR arms [71]. To estimate the substitution rate between the flanking LTR repeats, 5' and 3' repeats of each LTR retrotransposon predicted from LTRharvest were aligned by MUSCLE using default parameters provided in MEGA 5. After all gaps were removed, both number of differences and substitution rate were estimated in MEGA5. For number of differences, transitions and transversions were both included with a uniform rate. Substitution rate was modeled using the Kimura 2parameter model provided in MEGA5, and the rate variation among sites was modeled with a gamma distribution (shape parameter $=1$ ). The time since transposition was estimated based on the mutation rate previously reported for Populus tremula $\left(2.5 \times 10^{-9}\right.$ per year) [29].

\section{Detection of gene conversion}

As evidence of gene conversion, we searched for regions that were differentiated between species but concordant among the palindrome arms [31]. To accomplish this, we first aligned paired-end reads from a female clone of S. suchowensis (srx1561933) to the 94006 v5 female reference, plus alternative haplotypes, using Bowtie2 with the --local flag. This yielded an $82.9 \%$ overall alignment rate on average. The Illumina reads described above for clone 94006 were mapped using identical parameters. All reads aligning to the palindromes were extracted and compared to the whole genome using blastn. Mismapped reads originating from the autosomes were manually identified by scrutinizing the alignments, and only reads that mapped exclusively to the palindromic regions were retained. These reads were then re-aligned to a new reference consisting exclusively of arm 1 of the S. purpurea palindrome. SNPs and indels were called using mpileup and filtered to exclude loci with a minimum site quality $<$ Q20 or depth $>300$.

\section{Expression profiling}

RNAseq data was obtained from catkins of 10 female and 10 male $F_{2}$ progeny. RNAseq data were also obtained from multiple tissues of clones 94006 and Fish Creek. All sequences were Illumina $2 \times 150$ bp reads, except for 94006 , which were $2 \times 76$ bp reads. Transcripts from the palindrome can have high sequence identity among arms and with other paralogous sequences on the autosomes, which can complicate estimation of gene expression. Thus, all predicted coding sequences from the same gene family in the palindrome were aligned to the autosomal paralogs, and conserved sequences were masked in the reference genome. Salmon-0.11.3 [72] was used to quantify (salmon quant) the raw read count for each sample mentioned above with the gcBias flag as suggested by the developers. Heatmaps were generated separately for each group of palindrome genes, using $\log _{2}$ transformed data normalized with respect to library size or by variance stabilizing transformations (VST) using the R packages pheatmap and Deseq2 [73].

\section{Supplementary information}

Supplementary information accompanies this paper at https://doi.org/10. 1186/s13059-020-1952-4.

Additional file 1 Supplementary tables.

Additional file $\mathbf{2}$ Supplementary figures.

Additional file $\mathbf{3}$ Review history.

\section{Acknowledgements}

We thank Fred Gouker and the teams at the New York State Agricultural Experiment Station and West Virginia University for help with management and phenotyping of the field trials used in this study. We also thank the teams at Phytozome and JGl for facilitating public distribution of the data. We also thank Jennifer Hawkins, Niels Müller, and Mathias Fladung for helpful discussions.

Review history

The review history is available as Additional file 3.

Peer review information

Anahita Bishop was the primary editor on this article and managed its editorial process and peer review in collaboration with the rest of the editorial team.

\section{Funding}

This work was supported by the NSF Dimensions of Biodiversity Program (DEB-1542509 to S.D., DEB-1542486 to LBS, and DEB-1542599 to M.O.). Support was also provided by the National Natural Science Foundation of China (31590821, 31561123001, 31500502, 41871044), National Key Research and Development Program of China (2017YFC0505203, 2016YFD0600101), and the National Key Project for Basic Research (2012CB114504). The work conducted by the U.S. Department of Energy Joint Genome Institute is supported by the Office of Science of the U.S. Department of Energy under Contract No. DE-AC02-05CH11231.

\section{Availability of data and materials}

All sequence data used in this manuscript have been deposited in the NCBI Sequence Read Archive (https://www.ncbi.nlm.nih.gov/sra). Accession numbers are available in Additional file 1: Table S13. The genome assemblies and annotations are available through Phytozome (https://phytozome-next. jgi.doe.gov).

\section{Authors' contributions}

JS and JWJ assembled the genome; RZ and DM-S assembled and analyzed the W chromosome; DK, AS, LS, GAT, CC, and KB sequenced the genome and transcriptomes; SS annotated the genome; SD, LBS, TM, JL, and MO designed and led the study. SD, RZ, and DM-S wrote the manuscript. All authors read and approved the final manuscript.

Ethics approval and consent to participate Not applicable.

Consent for publication Not applicable.

Competing interests

The authors declare that they have no competing interests. 


\section{Publisher's Note}

Springer Nature remains neutral with regard to jurisdictional claims in published maps and institutional affiliations.

\begin{abstract}
Author details
'Department of Biology, West Virginia University, Morgantown, WV 26506-6057, USA. ${ }^{2}$ Horticulture Section, School of Integrative Plant Science, Cornell University, New York State Agricultural Experiment Station, Geneva, NY 14456, USA. ${ }^{3}$ HudsonAlpha Institute of Biotechnology, Huntsville, AL, USA. ${ }^{4}$ Department of Energy Joint Genome Institute, Walnut Creek, California, USA. ${ }^{5}$ Arizona Genomics Institute, School of Plant Sciences, University of Arizona, Tucson, AZ, USA. 'Biosciences Division, Oak Ridge National Laboratory, Oak Ridge, TN 37831, USA. ${ }^{7}$ DOE-Center for Bioenergy Innovation (CBI), Oak Ridge National Laboratory, Oak Ridge, TN 37831, USA. ${ }^{8}$ Key Laboratory of Bio-Resource and Eco-Environment of Ministry of Education, College of Life Sciences, Sichuan University, Chengdu 610065, China. ${ }^{9}$ State Key Laboratory of Grassland Agro-Ecosystem, Institute of Innovation Ecology \& College of Life Sciences, Lanzhou University, Lanzhou 730000, China. ${ }^{10}$ Department of Biological Sciences, Texas Tech University, Box 43131, Lubbock, TX 79409-3131, USA.
\end{abstract}

Received: 23 July 2019 Accepted: 3 February 2020 Published online: 14 February 2020

\section{References}

1. Bachtrog D. Y-chromosome evolution: emerging insights into processes of Y-chromosome degeneration. Nat Rev Genet. 2013;14:113-24.

2. Charlesworth D. Plant sex chromosome evolution. J Exp Bot. 2013;64:405-20

3. Charlesworth B, Charlesworth D. A model for the evolution of dioecy and gynodioecy. Am Nat. 1978;112:975-97

4. Skaletsky H, Kuroda-Kawaguchi T, Minx PJ, Cordum HS, Hillier L, Brown LG, et al. The male-specific region of the human $Y$ chromosome is a mosic of discrete sequence classes. Nature. 2003:423:825-37.

5. Betrán E, Demuth JP, Williford A. Why chromosome palindromes? Int J Evol Biol. 2012;2012:207958.

6. Trombetta B, Cruciani F. Y chromosome palindromes and gene conversion Hum Genet. 2017:136:605-19.

7. Navarro-Costa P, Plancha CE, Gonçalves J. Genetic dissection of the AZF regions of the human $Y$ chromosome: thriller or filler for male (in)fertility? J Biomed Biotechnol. 2010;2010:1-18.

8. Krausz C, Casamonti E. Spermatogenic failure and the $Y$ chromosome. Hum Genet. 2017:136:637-55

9. Warburton PE, Giordano J, Cheung F, Gelfand Y, Benson G. Inverted repeat structure of the human genome: the X-chromosome contains a preponderance of large, highly homologous inverted repeated that contain testes genes. Genome Res. 2004:14:1861-9.

10. Davis JK, Thomas PJ, Thomas JW. AW-linked palindrome and gene conversion in New World sparrows and blackbirds. Chromosom Res. 2010; 18:543-53

11. Ming R, Bendahmane A, Renner SS. Sex chromosomes in land plants. Annu Rev Plant Biol. 2011;62:485-514

12. Bachtrog D, Mank JE, Peichel CL, Kirkpatrick M, Otto SP, Ashman T-L, et al. Sex determination: why so many ways of doing it? PLoS Biol. 2014;12: e1001899.

13. Henry IM, Akagi T, Tao R, Comai L. One hundred ways to invent the sexes: theoretical and observed paths to dioecy in plants. Annu Rev Plant Biol. 2018;69:553-75.

14. Wang J, Na J, Yu Q, Gschwend AR, Han J, Zeng F, et al. Sequencing papaya $X$ and $Y^{h}$ chromosomes reveals molecular basis of incipient sex chromosome evolution. Proc Natl Acad Sci. 2012;109:13710-5.

15. Akagi T, Henry IM, Kawai T, Comai L, Tao R. Epigenetic regulation of the sex determination gene MeGI in polyploid persimmon. Plant Cell. 2016;28:2905-15.

16. Harkess A, Zhou J, Xu C, Bowers JE, Van der Hulst R, Ayyampalayam S, et al. The asparagus genome sheds light on the origin and evolution of a young Y chromosome Nat Commun. 2017:8:1279.

17. Tennessen JA, Wei N, Straub SCK, Govindarajulu R, Liston A, Ashman T-L. Repeated translocation of a gene cassette drives sex-chromosome turnover in strawberries. PLoS Biol. 2018;16:e2006062

18. Westergaard $M$. The mechanism of sex determination in dioecious flowering plants. Adv Genet. 1958;9:217-81.
19. Balounova V, Gogela R, Cegan R, Cangren P, Zluvova J, Safar J, et al Evolution of sex determination and heterogamety changes in section Otites of the genus Silene. Sci Rep. 2019;9:1045.

20. Zhou R, Macaya-Sanz D, Rodgers-Melnick E, Carlson CH, Gouker FE, Evans $L M$, et al. Characterization of a large sex determination region in Salix purpurea L. (Salicaceae). Mol. Genet. Genomics. 2018:293:1437-52.

21. Pucholt $P$, Rönnberg-Wästljung A-C, Berlin S. Single locus sex determination and female heterogamety in the basket willow (Salix viminalis L.). Heredity. 2015;114:575-83.

22. Hou J, Ye N, Zhang D, Chen Y, Fang L, Dai X, et al. Different autosomes evolved into sex chromosomes in the sister genera of Salix and Populus. Sci Rep. 2015;5:9076.

23. Tuskan GA, DiFazio S, Faivre-Rampant P, Gaudet M, Harfouche A, Jorge V, et al. The obscure events contributing to the evolution of an incipient sex chromosome in Populus: a retrospective working hypothesis. Tree Genet Genomes. 2012;8:559-71

24. Geraldes A, Hefer CA, Capron A, Kolosova N, Martinez-Nuñez F, Soolanayakanahally RY, et al. Recent Y chromosome divergence despite ancient origin of dioecy in poplars (Populus). Mol Ecol. 2015:24(13):3243-56.

25. Carlson CH, Choi Y, Chan AP, Serapiglia MJ, Town CD, Smart LB. Dominance and sexual dimorphism pervade the Salix purpurea $\mathrm{L}$. transcriptome. Genome Biol Evol. 2017;9:2377-94.

26. McKown AD, Klápště J, Guy RD, Soolanayakanahally RY, La Mantia J, Porth I, et al. Sexual homomorphism in dioecious trees: extensive tests fail to detect sexual dimorphism in Populus. Sci Rep. 2017;7:1831.

27. Bergero $R$, Charlesworth $D$. The evolution of restricted recombination in sex chromosomes. Trends Ecol Evol. 2009;24:94-102

28. van Doorn GS, Kirkpatrick M. Turnover of sex chromosomes induced by sexual conflict. Nature. 2007;449:909-12

29. Ingvarsson PK. Multilocus patterns of nucleotide polymorphism and the demographic history of Populus tremula. Genetics. 2008;180:329-40.

30. Hou J, Ye N, Dong Z, Lu M, Li L, Yin T. Major chromosomal rearrangements distinguish willow and poplar after the ancestral "Salicoid" genome duplication. Genome Biol Evol. 2016:8:1868-75.

31. Rozen S, Skaletsky H, Marszalek JD, Minx PJ, Cordum HS, Waterston RH, et al. Abundant gene conversion between arms of palindromes in human and ape $Y$ chromosomes. Nature. 2003;423:873-6.

32. Hughes JF, Skaletsky H, Pyntikova T, Graves TA, Van Daalen SKM, Minx PJ, et al. Chimpanzee and human y chromosomes are remarkably divergent in structure and gene content. Nature. 2010;463:536-9.

33. Hughes JF, Skaletsky H, Brown LG, Pyntikova T, Graves T, Fulton RS, et al. Strict evolutionary conservation followed rapid gene loss on human and rhesus Y chromosomes. Nature. 2012:483:82-6.

34. Soh YQS, Owens E, Brown LG, Alföldi J, Fulton RS, Wilson RK, et al. Sequencing the mouse $Y$ chromosome reveals convergent gene acquisition and amplification on both sex chromosomes. Cell. 2014;159:800-13.

35. Charlesworth D. Plant sex chromosomes. Annu Rev Plant Biol. 2016;67: 397-420.

36. Ming R, Moore PH. Genomics of sex chromosomes. Curr Opin Plant Biol. 2007;10:123-30

37. Bachtrog D, Mahajan S, Bracewell R. Massive gene amplification on a recently formed Drosophila Y chromosome. Nat Ecol Evol Nature Publishing Group. 2019:3:1587-97.

38. Scotti I, Delph LF. Selective trade-offs and sex-chromosome evolution in Silene latifolia. Evolution. 2006:60:1793-800.

39. Pandey RS, Azad RK. Deciphering evolutionary strata on plant sex chromosomes and fungal mating-type chromosomes through compositional segmentation. Plant Mol Biol. 2016;90:359-73.

40. Lange J, Skaletsky H, van Daalen SKM, Embry SL, Korver CM, Brown LG, et al. Isodicentric $Y$ chromosomes and sex disorders as byproducts of homologous recombination that maintains palindromes. Cell. 2009;138:855-69.

41. Goulding SE, Wolfe KH, Olmstead RG, Morden CW. Ebb and flow of the chloroplast inverted repeat. Mol Gen Genet. 1996;252:195-206.

42. Palmer JD, Thompson WF. Chloroplast DNA rearrangements are more frequent when a large inverted repeat sequence is lost. Cell. 1982;29:537-50.

43. Bellott DW, Hughes JF, Skaletsky H, Brown LG, Pyntikova T, Cho T-J, et al. Mammalian Y chromosomes retain widely expressed dosage-sensitive regulators. Nature. 2014:508:494-9.

44. Bellott DW, Skaletsky H, Cho T-J, Brown L, Locke D, Chen N, et al. Avian W and mammalian $Y$ chromosomes convergently retained dosage-sensitive regulators. Nat Genet. 2017:49:387-94. 
45. Bräutigam K, Soolanayakanahally R, Champigny M, Mansfield S, Douglas C, Campbell MM, et al. Sexual epigenetics: gender-specific methylation of a gene in the sex determining region of Populus balsamifera. Sci Rep. 2017;7: 45388.

46. Melnikova NV, Kudryavtseva AV, Borkhert EV, Pushkova EN, Fedorova MS, Snezhkina AV, et al. Sex-specific polymorphism of MET1 and ARR17 genes in Populus $\times$ sibirica. Biochimie. 2019;162:26-32.

47. Chefdor F, Héricourt F, Koudounas K, Carqueijeiro I, Courdavault V, Mascagni $F$, et al. Highlighting type a RRs as potential regulators of the dkHK1 multistep phosphorelay pathway in Populus. Plant Sci. 2018;277:68-78.

48. Ramírez-Carvajal GA, Morse AM, Davis JM. Transcript profiles of the cytokinin response regulator gene family in Populus. New Phytol. 2008;177:77-89.

49. Reyes-Olalde Jl, Zúñiga-Mayo VM, Serwatowska J, Chavez Montes RA, Lozano-Sotomayor P, Herrera-Ubaldo $\mathrm{H}$, et al. The bHLH transcription factor SPATULA enables cytokinin signaling, and both activate auxin biosynthesis and transport genes at the medial domain of the gynoecium. PLoS Genet. 2017;13:e1006726.

50. Wybouw B, De Rybel B. Cytokinin - a developing story. Trends Plant Sci. 2019:24:177-85

51. Akagi T, Henry IM, Ohtani H, Morimoto T, Beppu K, Kataoka I, et al. A Yencoded suppressor of feminization arose via lineage-specific duplication of a cytokinin response regulator in kiwifruit. Plant Cell. 2018;30:780-95.

52. Renner SS. The relative and absolute frequencies of angiosperm sexual systems: dioecy, monoecy, gynodioecy, and an updated online database. Am J Bot. 2014;101:1588-96.

53. Renner SS. Pathways for making unisexual flowers and unisexual plants: moving beyond the "two mutations linked on one chromosome" model. Am J Bot. 2016;103:587-9.

54. Chin C-S, Peluso P, Sedlazeck FJ, Nattestad M, Concepcion GT, Clum A, et al. Phased diploid genome assembly with single-molecule real-time sequencing. Nat Methods. 2016;13:1050-4.

55. Chin C-S, Alexander DH, Marks P, Klammer AA, Drake J, Heiner C, et al. Nonhybrid, finished microbial genome assemblies from long-read SMRT sequencing data. Nat Methods. 2013;10:563-9.

56. McKenna A, Hanna M, Banks E, Sivachenko A, Cibulskis K, Kernytsky A, et al. The genome analysis toolkit: a MapReduce framework for analyzing nextgeneration DNA sequencing data. Genome Res. 2010;20:1297-303.

57. Carlson CH, Gouker FE, Crowell CR, Evans L, SP DF, Smart CD, et al. Joint linkage and association mapping of complex traits in shrub willow (Salix purpurea L.). Ann Bot. 2019;124(4):701-716.

58. Langmead B, Salzberg SL. Fast gapped-read alignment with bowtie 2. Nat Methods. 2012;9:357-9.

59. Li H, Handsaker B, Wysoker A, Fennell T, Ruan J, Homer N, et al. The sequence alignment/map format and SAMtools. Bioinformatics. 2009;25: 2078-9.

60. Catchen J, Hohenlohe PA, Bassham S, Amores A, Cresko WA. Stacks: an analysis tool set for population genomics. Mol Ecol. 2013;22:3124-40.

61. Margarido GR, Souza AP, Garcia AA. OneMap: software for genetic mapping in outcrossing species. Hereditas. 2007;144:78-9.

62. Tang H, Zhang X, Miao C, Zhang J, Ming R, Schnable JC, et al. ALLMAPS: robust scaffold ordering based on multiple maps. Genome Biol. 2015;16:3.

63. Wang Y, Tang H, DeBarry JD, Tan X, Li J, Wang X, et al. MCScanX: a toolkit for detection and evolutionary analysis of gene synteny and collinearity. Nucleic Acids Res. 2012;40:e49.

64. Wilm A, Higgins DG, Valentin F, Blackshields G, McWilliam H, Wallace IM, et al. Clustal W and Clustal X version 2.0. Bioinformatics. 2007;23:2947-8.

65. Yang Z. PAML 4: phylogenetic analysis by maximum likelihood. Mol Biol Evol. 2007;24:1586-91.

66. Gouker FE, DiFazio SP, Bubner B, Zander M, Smart LB. Genetic diversity and population structure of native, naturalized, and cultivated Salix purpurea. Tree Genet Genomes. 2019;15:47.

67. Kang HM, Sul JH, Service SK, Zaitlen NA, Kong SY, Freimer NB, et al. Variance component model to account for sample structure in genome-wide association studies. Nat Genet. 2010;42:348-54

68. Tamura K, Peterson D, Stecher G, Peterson N, Kumar S, Nei M. MEGA5: molecular evolutionary genetics analysis using maximum likelihood, evolutionary distance, and maximum parsimony methods. Mol Biol Evol. 2011;28:2731-9.

69. Ellinghaus D, Kurtz S, Willhoeft U. LTRharvest, an efficient and flexible software for de novo detection of LTR retrotransposons. BMC Bioinformatics. 2008:9:18.
70. Steinbiss S, Willhoeft U, Gremme G, Kurtz S. Fine-grained annotation and classification of de novo predicted LTR retrotransposons. Nucleic Acids Res. 2009;37:7002-13.

71. SanMiguel P, Gaut BS, Tikhonov A, Nakajima Y, Bennetzen JL. The paleontology of intergene retrotransposons of maize. Nat Genet. 1998;20: 43-5.

72. Patro R, Duggal G, Love MI, Irizarry RA, Kingsford C. Salmon provides fast and bias-aware quantification of transcript expression. Nat Methods. 2017; 14:417-9.

73. Love Ml, Huber W, Anders S. Moderated estimation of fold change and dispersion for RNA-seq data with DESeq2. Genome Biol. 2014;15:550.

\section{Ready to submit your research? Choose BMC and benefit from:}

- fast, convenient online submission

- thorough peer review by experienced researchers in your field

- rapid publication on acceptance

- support for research data, including large and complex data types

- gold Open Access which fosters wider collaboration and increased citations

- maximum visibility for your research: over $100 \mathrm{M}$ website views per year

At BMC, research is always in progress.

Learn more biomedcentral.com/submissions 